\title{
Cerebellar-recipient motor thalamus drives behavioral context-specific movement initiation
}

\author{
Joshua Dacre ${ }^{1,2,}$, Matt Colligan*1,2, Julian Ammer ${ }^{\star 1,2}$, Julia Schiemann $^{1,2}$, Thomas Clarke ${ }^{1,2}$, \\ Victor Chamosa-Pino ${ }^{1,2}$, Federico Claudi ${ }^{1,2}$, J. Alex Harston ${ }^{1,2}$, Constantinos Eleftheriou ${ }^{1,2}$, \\ Janelle M.P. Pakan ${ }^{1,2}$, Cheng-Chiu Huang ${ }^{3}$, Adam Hantman ${ }^{3}$, Nathalie L. Rochefort ${ }^{1,2}$, Ian \\ Duguid $^{1,2,}$. \\ ${ }^{1}$ Centre for Discovery Brain Sciences and Patrick Wild Centre, Edinburgh Medical School: Biomedical Sciences, \\ University of Edinburgh, Edinburgh, EH8 9XD, UK. \\ ${ }^{2}$ Simons Initiative for the Developing Brain, University of Edinburgh, Edinburgh, EH8 9XD, UK. \\ ${ }^{3}$ Janelia Research Campus, HHMI, Ashburn, Virginia, 20147, USA \\ *These authors contributed equally to the work. \\ Corresponding authors: jdacre@ed.ac.uk, lan.Duguid@ed.ac.uk
}

Address for editorial correspondence:

lan Duguid

Centre for Discovery Brain Sciences

University of Edinburgh

Edinburgh Medical School: Biomedical Sciences

Hugh Robson Building

George Square

Edinburgh

EH8 9XD, UK

Tel. +44131650 3113

Email: lan.Duguid@ed.ac.uk 


\section{Summary}

To initiate goal-directed behavior, animals must transform sensory cues into motor commands that generate appropriately timed actions. Sensorimotor transformations along the cerebellarthalamocortical pathway are thought to shape motor cortical output and movement timing, but whether this pathway initiates goal-directed movement remains poorly understood. Here, we recorded and perturbed activity in cerebellar-recipient regions of motor thalamus (dentate / interpositus nucleus-recipient regions, $\left.\mathrm{MTh}_{\mathrm{DN} / \mathrm{IPN}}\right)$ and primary motor cortex (M1) in mice trained to execute a cued forelimb lever push task for reward. MTh $h_{D N / P N}$ population responses were dominated by a time-locked increase in activity immediately prior to movement that was temporally uncoupled from cue presentation, providing a fixed latency feedforward motor timing signal to $M 1_{\mathrm{FL}}$. Blocking $\mathrm{MTh} \mathrm{h}_{\mathrm{DN} / \mathrm{IPN}}$ output suppressed cued movement initiation. Stimulating the MTh $\mathrm{DNN}_{\mathrm{PN}}$ thalamocortical pathway in the absence of the cue recapitulated cueevoked M1 membrane potential dynamics and forelimb behavior in the learned behavioral context, but generated semi-random movements in an altered behavioral context. Thus, cerebellar-recipient motor thalamocortical input to M1 is indispensable for the generation of motor commands that initiate goal-directed movement, refining our understanding of how the cerebellar-thalamocortical pathway contributes to movement timing.

\section{Introduction}

The ability to generate precisely timed motor actions in response to sensory cues is a hallmark of mammalian motor control. Movement timing is believed to be mediated by cerebellumdependent shaping of motor output (Holmes, 1939) given that damage to, or inactivation of, the cerebellum results in poorly timed motor actions (Bastian and Thach, 1995; Milak et al., 1997; Thach, 1975). However, the circuit mechanisms that generate motor timing signals necessary for goal-directed movement initiation remain unclear. Cerebellar control of goaldirected movement is predominantly mediated via two distinct pathways, the cerebellarrubrospinal tract (Gibson et al., 1985) and the more dominant cerebellar-thalamocortical pathway (Horne and Butler, 1995). Feedforward excitatory input from the deep cerebellar 
nuclei (DCN) provides one of the main driver inputs to the motor thalamus and is thought to be necessary for controlling the timing of simple and complex movements (Ivry and Keele, 1989; Mink and Thach, 1991; Ohmae et al., 2017). But whether the cerebello-thalamocortical pathway is required for movement initiation has been much debated (Thach, 2013). Neuronal activity in the dentate and interpositus subdivisions of the deep cerebellar nuclei and their recipient regions in motor thalamus precedes activity changes in motor cortex and movement (Bosch-Bouju et al., 2014; Butler et al., 1992; Fortier et al., 1989; Harvey et al., 1979; Mushiake and Strick, 1993; Thach, 1975, 1978), suggestive of a role in movement initiation. However, local inactivation of dentate and interpositus nuclei or their recipient regions in motor thalamus during simple cued forelimb tasks produces variable behavioral outcomes, from no effect (Miller and Brooks, 1982) to slowing of reaction times (Meyer-Lohmann et al., 1977; Mink and Thach, 1991; Spidalieri et al., 1983; Thach, 1975) and reduced task engagement (van Donkelaar et al., 2000). Although suggestive of a role in movement timing, direct causal evidence supporting a role for the cerebellar-thalamocortical pathway in movement initiation has been lacking.

If the cerebellar-thalamocortical pathway conveys motor timing signals, $M T h_{D N / P N}$ activity could be described by three hypothetical models. Either, (i) population activity rises from a fixed timepoint prior to movement initiation which is temporally uncoupled from cue onset (i.e. a motor timing signal that has a fixed onset and fixed slope when aligned to movement); (ii) population responses rise from cue onset to a timepoint distant from movement initiation (i.e. variable onset, fixed slope trajectories where $M M_{\text {DN/IPN }}$ input does not directly correlate with movement initiation); or (iii) MTh directly coupled to movement initiation (i.e. linear sensorimotor transformation from cue to movement, variable onset, variable slope trajectories) (Figures 1a and 1b).

To distinguish between these models and to investigate whether the cerebellar-recipient motor thalamocortical pathway is necessary for goal-directed movement initiation, we employed 
thalamic population calcium imaging, patch-clamp recordings in $M 1$, and targeted manipulations in mice trained to execute a cued forelimb push task for reward. We demonstrate that $M T h_{D N / I N N}$ population responses were dominated by a time-locked increase in activity immediately prior to movement initiation that was temporally uncoupled from cue presentation, providing a fixed latency feedforward motor timing signal to $\mathrm{M}_{\mathrm{FL}}$. Focal inactivation of MTh DN/IPN suppressed layer 5 membrane potential dynamics in forelimb motor cortex $\left(\mathrm{M} 1_{\mathrm{FL}}\right)$ and blocked cued movement initiation. Direct stimulation of $\mathrm{MTh}_{\mathrm{DN} / \mathrm{PN}}$ neurons, or their axon terminals in $\mathrm{M}_{\mathrm{FL}}$, in the absence of the cue recapitulated motor cortical activity dynamics and forelimb behavior in the learned behavioral context, but generated semi-random movements in an altered behavioral context where the lever and reward were absent. Together, our findings demonstrate that the cerebellar-recipient motor thalamocortical pathway conveys essential motor timing signals necessary for the initiation of goal-directed movement.

\section{Results}

To explore the role of MTh linear forelimb push task for mice. The design of the task, which incorporates a horizontal translation lever, required mice to learn the correct wrist and grip orientation to ensure smooth, friction-reduced horizontal lever movements $(4 \mathrm{~mm})$ in response to a $6 \mathrm{kHz}$ auditory cue (Figure $1 \mathrm{c}$ and Video S1). Mice rapidly learned to execute the task (mean $=7.5$ days, $95 \% \mathrm{Cl}$ $[6.3,8.6], N=16$ mice, all data, unless otherwise stated, are presented as mean, [bootstrapped 95\% confidence interval]; last session task success, mean $=0.64$ rewards per cue presentation, $95 \% \mathrm{Cl}[0.56,0.72]$ ), displaying relatively fast reaction times (last session, median $=0.32 \mathrm{~s}[0.30,0.34])$ and reproducible forelimb kinematic trajectories (Figures $1 \mathrm{~d}-1 \mathrm{f}$, Video S1). 
a

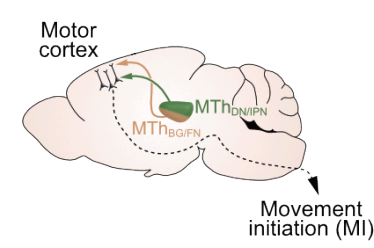

b

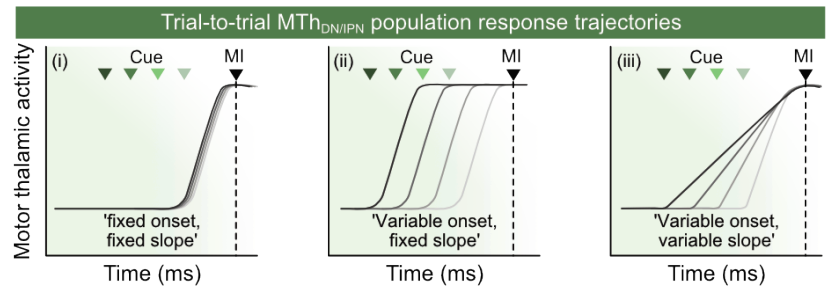

d

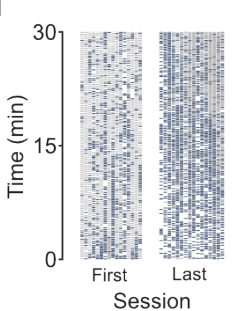

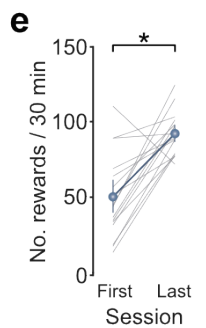

f

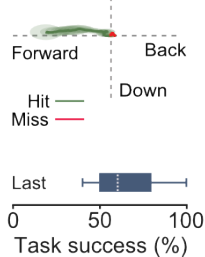

$2 \mathrm{~mm} \quad$ Up g

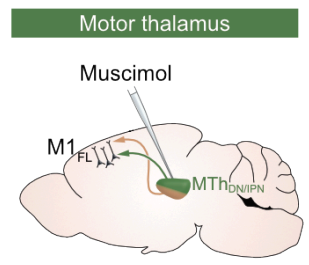

h

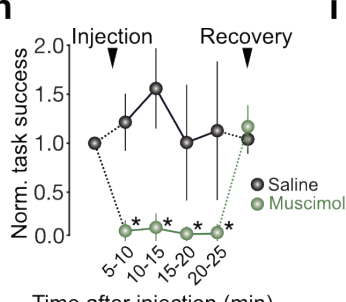

Time after injection (min) i

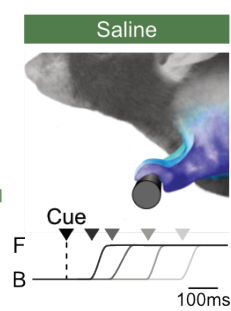

j

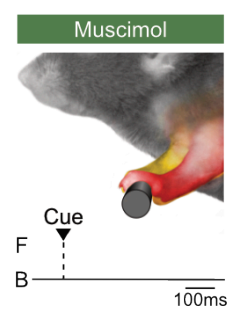

Figure 1. Motor thalamic output is necessary for cued goal-directed movement initiation in mice.

(a) Sagittal mouse brain schematic depicting feedforward input from dentate / interpositus nucleus-recipient (MTh $\mathrm{DNN}_{\mathrm{PN}}$ ) and basal ganglia / fastigial nucleusrecipient $\left(M T h_{B G / F N}\right.$ ) regions of motor thalamus to motor cortex. (b) Hypothetical trial-to-trial $M \mathrm{Mh}_{\mathrm{DN} / \mathrm{PN}}$ population response trajectories: model (i), fixed onset, fixed slope; model (ii), variable onset, fixed slope; model (iii), variable onset, variable slope. Green triangles depict cue onset across 4 trials, response trajectories are aligned to movement initiation (Ml, black triangle and dashed line). (c) Top, cued goal-directed linear forelimb push task for head restrained mice. Ml, movement initiation. Bottom, behavioral task structure: ITI, inter-trial interval; IGM, internally generated movement. (d) Left \& right, rasters showing behavioral task success across first and last training sessions. Each column represents the behaviour of an individual mouse across the training session $(\mathrm{N}=16)$. Blue, hit trials; grey, miss trials; white, IGMs. (e) Task metrics across learning. Left, average number of rewards received per 30 minutes $\left(\mathrm{N}=16 \mathrm{mice}, t(15)=-5.3\right.$, ${ }^{*} P=9.5 \times 10^{-5}$, twosample $t$-test). Right, box-and-whisker plots show median, interquartile range and range of median reaction times (RTs) across mice on the first and last day of training $\left(N=16\right.$ mice, $t(15)=7.1,{ }^{*}=3.4 \times 10^{-6}$, two-sample $t$-test). (f) Top, average kinematic forepaw trajectory for hit (green) and miss (red) trials from an example mouse shown in Supp. Video 2 . Thick line depicts average trajectory overlaid with the $95 \% \mathrm{Cl}$ of frame-by-frame paw position variance (transparent ovals). Bottom, box-and-whisker plot showing median, interquartile range and range of task success across mice during last training session. (g) Focal muscimol inactivation of thalamus, targeting $\mathrm{MTh}_{\mathrm{DN} \text { IIPN. }}$ (h) Normalised task success as a function of time after muscimol injection. Colored symbols represent changes in population means $\pm 95 \% \mathrm{Cl}$ after saline (black, $\mathrm{N}=5$ mice) or muscimol (green, $\mathrm{N}=5$ mice) injection $\left(5-10\right.$ minute bin, $F(1,8)=63.0$, ${ }^{*} P=1.9 \times 10^{-4}, 2$-way repeated measures ANOVA, with a Bonferroni-Holm correction for multiple comparisons). (i-j) Top: Superimposed images of mouse forelimb position at cue onset

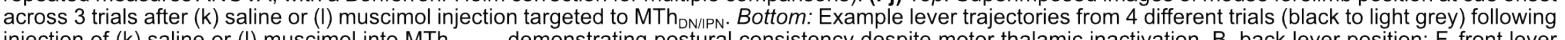
injection of $(k)$ saline or (l) muscimol into $M T^{D N / P N}$, demonstrating postural consistency despite motor thalamic inactivation. $B$, back lever position; $F$, front lever position; black triangle and dashed line represent cue presentation; grey triangles indicate reaction times.

To selectively record and manipulate MThDN/PN activity during behavior, we confirmed the anatomical location of thalamic nuclei that send monosynaptic projections to $\mathrm{M} 1_{\mathrm{FL}}$ and receive dense projections from the dentate (DN) and interpositus (IPN) deep cerebellar nuclei (Gao et al., 2018; Kuramoto et al., 2009; Rispal-Padel et al., 1987; Sakai et al., 1996; Schiemann et al., 2015). By employing conventional retrobead fluorescence tracing across layers 2-6 in $\mathrm{M} 1_{\mathrm{FL}}$, layer 5-specific monosynaptic rabies tracing and anterograde viral tracing from the DN and IPN, we observed dense expression in dorsal-posterior motor thalamus centered on the ventrolateral nucleus $(\mathrm{VL})$ with expression in anteromedial $(\mathrm{AM})$, ventral posteromedial (VPM) and ventral posterolateral (VPL) nuclei, but no staining in the ventromedial nucleus (VM), which primarily receives input from the basal ganglia (BG) and fastigial nucleus (FN) (Figure 
1a, Figures S1 and S2) (Kuramoto et al., 2009; Person et al., 1986; Sakai et al., 1996; Tanaka et al., 2018). Moreover, the vast majority of neurons in MTh $\mathrm{M} 1_{\mathrm{FL}}$ highlighting the high degree of connectivity between two important nodes along the cerebellar-thalamocortical pathway $($ mean $=76.0 \%, 95 \% \mathrm{Cl}[69.4,82.8], \mathrm{n}=16$ slices from $\mathrm{N}$ $=3$ mice, Figure S3).

To investigate whether MTh $h_{D N / P N}$ was necessary for cued goal-directed movement initiation, we focally injected a small bolus of the $\mathrm{GABA}_{A}$ receptor agonist muscimol centered on the ventrolateral nucleus, with an estimated spread of $\sim 500 \mu \mathrm{m}$ from the point of injection within 10 minutes (Martin, 1991) (Figure 1g and Figure S4). By applying muscimol during behavioral task engagement, we recorded the immediate post-injection effects. Muscimol reduced task success by $\sim 90 \%, 5-10$ minutes after injection, an effect that persisted for the duration of the session before reverting to baseline after 24 hours $(5-10$ mins, mean $=0.11$ normalized task success, $95 \% \mathrm{Cl}\left[0.04,0.18, \mathrm{~N}=5\right.$ mice, $F(1,8)=63.0, P=1.9 \times 10^{-4}$, two-way ANOVA with Bonferroni-Holm correction for multiple comparisons) (Figure 1h). Reduced task success was not a result of task disengagement as cue presentation reproducibly evoked short-latency whisking and enhanced arousal (Video S2). Moreover, mice did not experience a loss of forelimb postural control, as evidenced by the accurate trial-to-trial forelimb positioning at cue presentation (Figures $1 \mathrm{i}$ and $1 \mathrm{j}$, Figure S4). The predominant effect of $\mathrm{MTh}_{\mathrm{DN} / \mathrm{IPN}}$ inactivation was not a slowing of reaction times (Meyer-Lohmann et al., 1977; Mink and Thach, 1991; Spidalieri et al., 1983; Thach, 1975; van Donkelaar et al., 2000), instead it selectively blocked movement initiation. Given that $M T_{\mathrm{DN} / \mathrm{PN}}$ provides feedforward excitation to $\mathrm{M} 1_{\mathrm{FL}}$, we next assessed whether motor cortical output was also necessary by focally injecting muscimol into the center of $\mathrm{M} 1_{\mathrm{FL}}$ (Schiemann et al., 2015). $\mathrm{M}_{\mathrm{FL}}$ inactivation reduced task success by $\sim 70 \%$, 5-10 minutes after injection, persisting for the duration of the session before reverting to baseline after 24 hours (5-10 mins, mean $=0.29$ normalized task success, $95 \% \mathrm{Cl}[0.11,0.51]$, $\mathrm{N}=5$ mice, $F(1,8)=3.7, P=0.09$, two-way ANOVA with Bonferroni-Holm correction for multiple comparisons). Dissimilar to motor thalamic inactivation, silencing $\mathrm{M} 1_{\mathrm{FL}}$ output induced 
bioRxiv preprint doi: https://doi.org/10.1101/802124; this version posted October 16, 2019. The copyright holder for this preprint (which was not certified by peer review) is the author/funder, who has granted bioRxiv a license to display the preprint in perpetuity. It is made available under aCC-BY 4.0 International license.

a loss of forelimb postural control and hemiplegia, resulting in an inability to engage with the lever and task (Figure S4 and Video S3). Together, these data suggest that M1 is essential for coordinated motor control but MTh initiation in mice.

To test whether MTh ${ }_{D N / I P N}$ response timing was consistent with a role in movement initiation, we employed Gradient Refractive Index (GRIN) lens-mediated 2-photon population calcium imaging of MTh $h_{D N / P N}$ neurons during task engagement (Figures $2 a$ and $2 b$ ). We found that the majority of MTh ${ }_{D N / P N}$ neurons displayed task-related activity changes (192/248 neurons, 11 fields of view (FOV), $N=8$ mice) either prior to movement initiation (early onset positive $\Delta F / F_{0}$, $127 / 192$ neurons; early onset negative $\Delta F / F_{0}, 24 / 192$ neurons) or during the post-movement period (late onset positive $\Delta F / F_{0}, 18 / 192$ neurons; late onset negative $\Delta F / F_{0}, 23 / 192$ neurons) (Figures $2 \mathrm{c}$ and $2 \mathrm{~d}$ ). The most prominent activity profile of early onset neurons - i.e. activity that could contribute to movement initiation - was enhanced activity that occurred after the cue but $\sim 300$ ms prior to movement initiation (early onset neurons $-84.1 \%$ enhanced, $15.9 \%$ suppressed) (Figure 2d). This dominant population response profile was consistent across

a

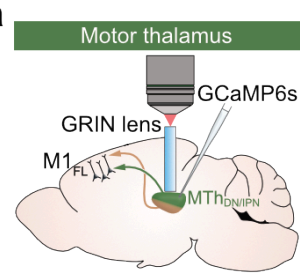

b

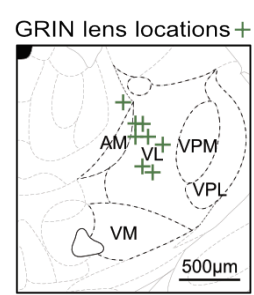

Bregma $-1.22 \mathrm{~mm}$
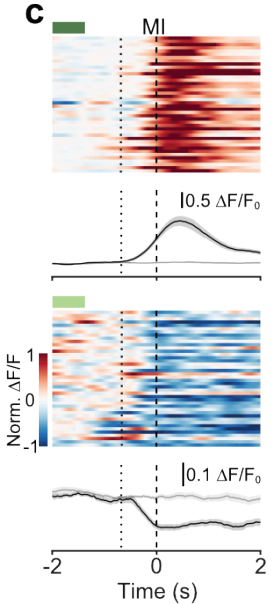
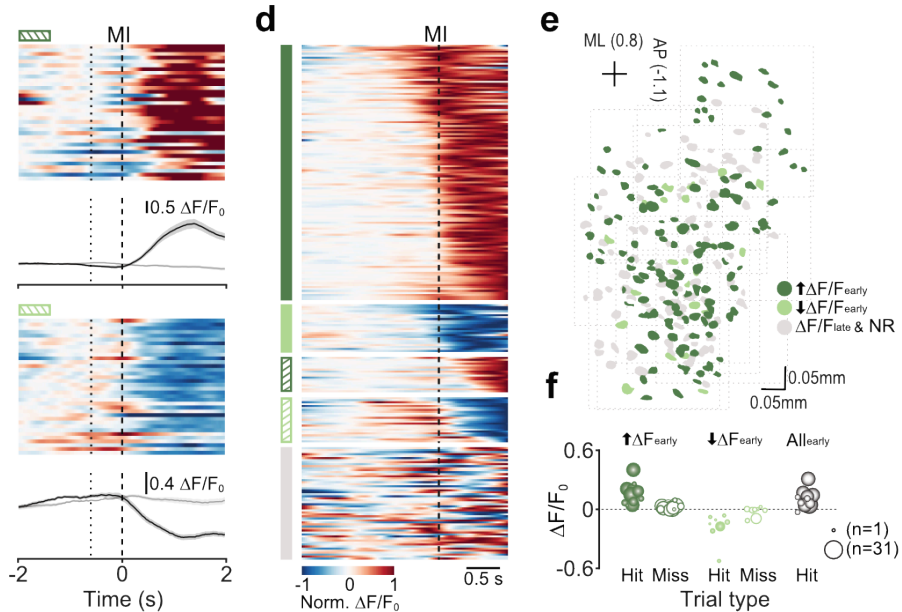
Trial type

Figure 2. Enhanced activity dominates trial-to-trial $M \mathrm{MT}_{\mathrm{DN} \text { NIPN }}$ population responses prior to movement initiation.

(a) Gradient-index (GRIN) lens-mediated 2-photon population calcium imaging in $M T h_{D N / P N}$. $M 1_{F L}$, forelimb motor cortex; $M T h_{D N / P N}$, dentate / interpositus nucleus-recipient region of motor thalamus. (b) Anatomical locations of GRIN lens placements in MTh VL, ventrolateral; VPM, ventral posteromedial nucleus; VPL, ventral posteromedial; VM, ventromedial. (c) Four example MTh DN/IPN neurons: clockwise from top left, 'early onset enhanced' (dark green), 'late onset enhanced' (dark green hatching), 'late onset suppressed' (light green hatching), 'early onset suppressed' (light green). Top, raster showing normalised $\Delta F / F_{0}$ across successive trials. Bottom, $\Delta F / F_{0}$ mean \pm s.e.m. Black lines represent hit trials, grey lines represent miss trials. Dotted lines, median cue onset; dashed lines, movement initiation (MI). (d) Raster showing average $\Delta F / F_{0}$ across trials for individual neurons Neurons are classified and grouped into 'early onset enhanced' (dark green, $n=127 / 248$ neurons); 'early onset suppressed' (light green, $n=24 / 248$ neurons) 'late onset enhanced' (dark green hatching, $n=18 / 248$ neurons); 'late onset suppressed' (light green hatching, $n=23 / 248$ neurons); and 'non-responsive' (grey, $n=56 / 248$ neurons). Neurons are ordered by $\Delta F / F_{0}$ onset $(n=11$ fields of view, $N=8$ mice). (e) Spatial distribution of early onset enhanced (dark green), early

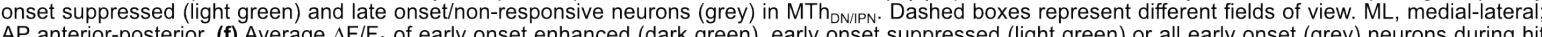
AP anterior-posterior. (f) Average $\Delta F / F_{0}$ of early onset enhanced (dark green), early onset suppressed (light green) or all early onset (grey) neurons during hit
(filled symbols) and miss trials (open symbols) separated by FOV. Circle size represents number of neurons per field of view [range $1-31$ ]. 
bioRxiv preprint doi: https://doi.org/10.1101/802124; this version posted October 16, 2019. The copyright holder for this preprint (which was not certified by peer review) is the author/funder, who has granted bioRxiv a license to display the preprint in perpetuity. It is made available under aCC-BY 4.0 International license.

trials and was not spatially restricted to a defined region of $M T_{\text {DN/PN }}$ (Figures 2c-2e). To investigate whether $\mathrm{MTh}_{\mathrm{DN} / \mathrm{IPN}}$ population responses conveyed information regarding the cue or a purely movement-related signal, we exploited miss trials where mice perceived the cue, as indicated by short-latency whisking and increased arousal, but did not engage in the task (see Video S1). In the absence of movement, no appreciable cue-evoked responses across early onset neurons were observed (Figures $2 c$ and 2f), suggesting MTh a purely motor-related feedforward signal.

We next tested which of our MTh trial activity in early onset enhanced neurons (see Figure 1b). Clustering trials by short, medium and long reaction times (RTs) and aligning averaged trial-to-trial population $\Delta \mathrm{F} / \mathrm{F}_{0}$ traces to movement initiation, $\mathrm{MTh}_{\mathrm{DN} / \mathrm{PN}}$ responses displayed a consistent, sharp increase in $\Delta \mathrm{F} / \mathrm{F}_{0} \sim 300 \mathrm{~ms}$ prior to movement initiation, irrespective of reaction time (short $\mathrm{RT}$, mean onset $=308 \mathrm{~ms}, 95 \% \mathrm{Cl}[248,377]$; medium RT, mean onset = $299 \mathrm{~ms}, 95 \% \mathrm{Cl}$ [205, 392]; long RT, mean onset $=351 \mathrm{~ms}, 95 \% \mathrm{Cl}[251,455], 117$ neurons, 9 fields of view (FOV), $\mathrm{N}=7$ mice, for

a

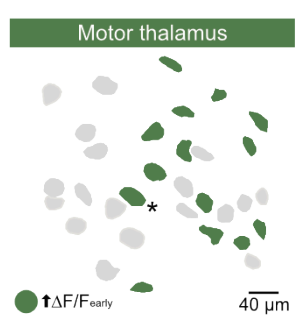

d

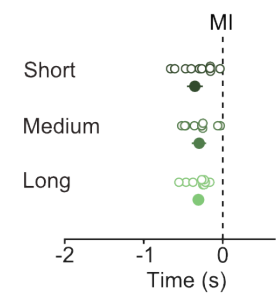

b

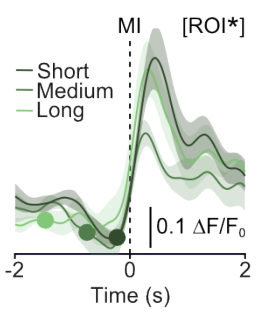

e

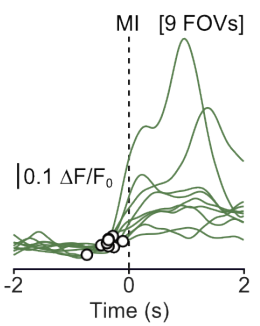

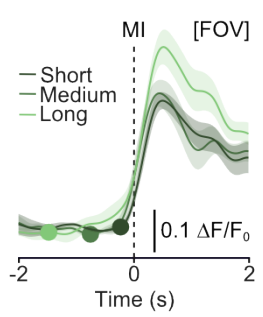

$\mathbf{f}$

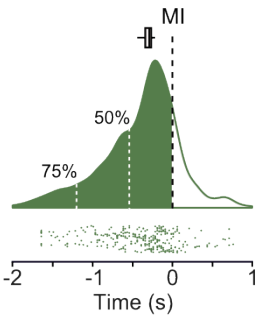

Figure 3. $M T h_{\text {DNIPN }}$ population responses provide a reliable trial-to-trial movement initiation signal.

(a) Spatial distribution of early onset enhanced neurons in a representative field of view in $M T h_{D N / P N}$. (b-c) Average $\Delta F / F_{0}$ from (b) a single early onset enhanced neuron depicted in panel (a) by $a^{*}$, or (c) all early onset enhanced neurons in the field of view shown in panel (a), aligned to movement initiation (MI) and split by short, medium and long reaction time trials. Colored circles depict median time of cue presentation. [ROI], region of interest; [FOV], field of view. Mean \pm s.e.m. (d) Motor thalamic population response trajectory onsets split by short, medium and long reaction times. Open circles represent individual fields of view, filled circles represent means $\pm 95 \% \mathrm{Cl}\left(n=9\right.$ fields of view, $N=7$ mice). Dashed line depicts movement initiation $(\mathrm{MI})$. (e) Average $\Delta \mathrm{F} / \mathrm{F}_{0}$ trajectories from nine early onset enhanced neurons from different fields of view (FOVs) with response onsets indicated by black circles. Dashed line depicts movement initiation (MI). (f) Raincloud plot showing the distribution of bootstrapped trial-to-trial motor thalamic response onsets for all early onset enhanced neurons across nine fields of view. Top, box-and-whisker plot of the median onset bootstrapped estimate. Middle, average kernel density estimation of trial-to-trial motor thalamic response onsets with $50 \%$ and $75 \%$ AUC to
movement initiation (MI) depicted by white vertical dashed lines. Bottom, raster of trial-to-trial early enhanced population onset times 
inclusion criteria see Methods) (Figures 3a-3d). Moreover, trial-to-trial consistency in MTh (Figures $3 e$ and $3 f)$. Thus, our results are consistent with a model whereby $M T h_{D N / I N N}$ output provides a reliable time-locked motor signal immediately prior to movement initiation that is temporally uncoupled from the sensory cue (i.e. model (i) in Figure 1b).

To gain a mechanistic insight into how feedforward input from MTh membrane potential dynamics, we performed whole-cell patch-clamp recordings from identified L5B projection neurons in $\mathrm{M} 1_{\mathrm{FL}}$ (Figure 4a). When aligned to movement initiation, L5B neurons displayed a continuum of subthreshold membrane potential changes, biased towards depolarizing $V_{m}$ (depolarizing, $n=15 / 23$ neurons; hyperpolarizing, $n=4 / 23$ neurons, non-responsive, $n=4 / 23, N=23$ mice), with the direction of the $\Delta V_{m}$ being consistent from trial-to-trial (Figures $4 \mathrm{~b}-4 \mathrm{f}$ and Figure S5). Importantly, peak-scaled $\mathrm{V}_{\mathrm{m}}$ traces - whether depolarizing or hyperpolarizing - displayed stereotyped trajectories where the $\Delta \mathrm{V}_{\mathrm{m}}$ onset closely matched the distribution of $M T_{D N / P N}$ population response onsets (i.e. $\sim 300 \mathrm{~ms}$ prior to movement initiation, Figures $4 \mathrm{~g}-4 \mathrm{i})$. To investigate whether $L 5 B V_{m}$ dynamics are driven entirely by input from motor thalamus, we again exploited miss trials in which thalamic population responses are absent (see Figures $2 c$ and $2 f$ ). $V_{m}$ trajectories were on average smaller in amplitude and duration (mean miss:hit AUC ratio $=0.65,95 \% \mathrm{Cl}[0.50,0.80]$ ), suggesting that convergence of thalamic and other long-range inputs is necessary for cued goal-directed movement initiation (Figure 4j and Figure S5). To drive movement, subthreshold $V_{m}$ changes must transform into behaviorally-relevant spiking. Accordingly, we found a strong correlation between changes in $\mathrm{V}_{\mathrm{m}}$ and firing rate across layer 5B projection neurons, including pyramidal tract (PT-type) neurons that have direct access to brainstem and spinal cord circuits controlling voluntary movement (Figures $4 \mathrm{k}-4 \mathrm{~m}$ and Figure S5). Next, we focally injected a small bolus of muscimol centered on the ventrolateral nucleus while recording from identified L5B projection neurons (Figure $4 \mathrm{n}$ ) to explore a causal link between thalamic input, L5B $V_{m}$ 
bioRxiv preprint doi: https://doi.org/10.1101/802124; this version posted October 16, 2019. The copyright holder for this preprint (which was not certified by peer review) is the author/funder, who has granted bioRxiv a license to display the preprint in perpetuity. It is made available under aCC-BY 4.0 International license.

a

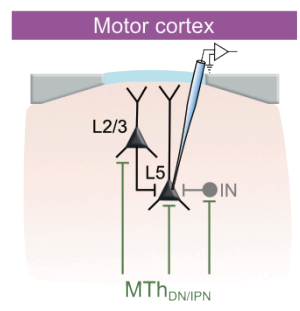

d

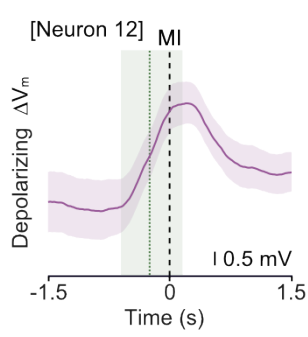

g

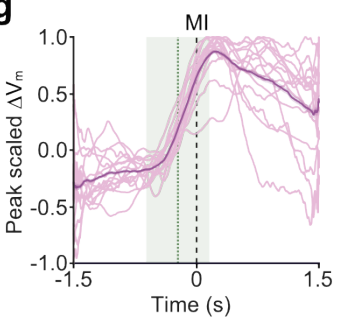

k

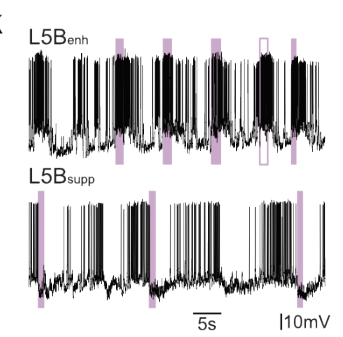

n

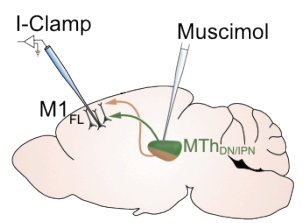

b

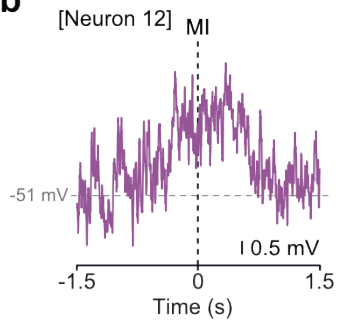

e

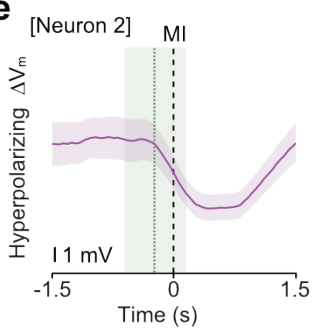

h

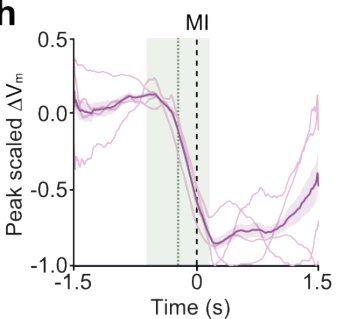

I

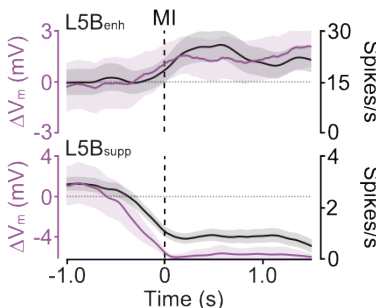

C [Neuron 2] MI

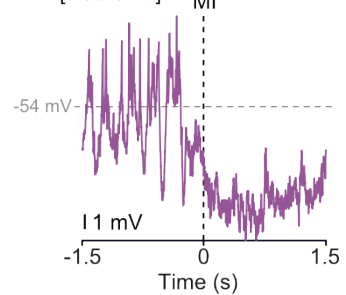

f
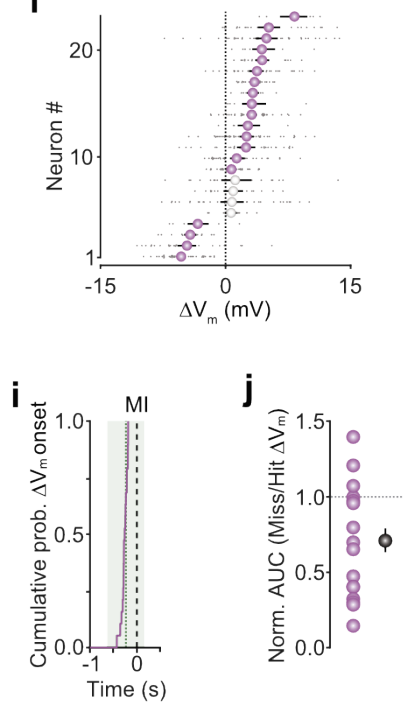

m

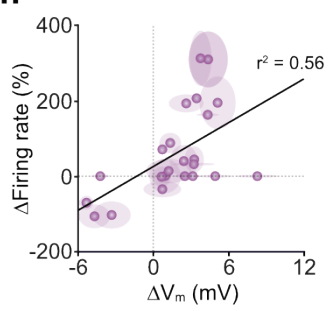

$\mathbf{0}$

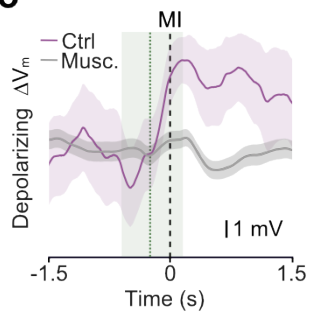

p

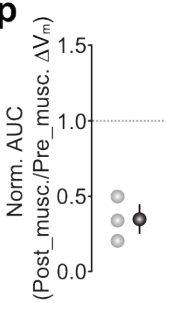

q

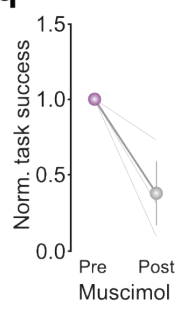

Figure 4. Feedforward $\mathrm{MTh}_{\mathrm{DNIPN}}$ input is necessary for bidirectional $\mathrm{M1}_{\mathrm{FL}}$ L5B output modulation and cued goal-directed movement initiation.

(a) Patch-clamp recording from L5B projection neurons in $M 1_{\mathrm{FL}}$. IN, interneuron; $M \mathrm{Th}_{\mathrm{DN} / \mathrm{IPN}}$, dentate / interpositus nucleus-recipient region of motor thalamus. (b-c) Representative single trial subthreshold membrane potential $\left(V_{m}\right)$ trajectories from two L5B projection neurons showing either a depolarization (b) or hyperpolarization (c) prior to movement initiation (MI). Spikes have been clipped to improve visualisation of the subthreshold $V_{m}$. (d-e) Average changes in subthreshold $\mathrm{V}_{\mathrm{m}} \pm 95 \% \mathrm{Cl}$ in the two L5B projection neurons depicted in (b) and (c). In these and subsequent figure panels, the green dashed line depicts mean $\mathrm{MTh}_{\text {DN/PN }}$ activity onset $\pm 95 \% \mathrm{Cl}$ as shown in Fig. $3 \mathrm{~d}$ and the black dashed line represents movement initiation (MI). (f) Average L5B projection neuron perimovement $\Delta \mathrm{V}_{m} \pm 95 \% \mathrm{Cl}$. Grey dots represent individual trials, purple symbols represent significant $\Delta \mathrm{V}_{m}$ changes, white symbols represent non-significant changes, defined by comparing $95 \%$ bootstrapped confidence intervals $\left(n=23\right.$ neurons, $N=23$ mice). (g-h) Peak scaled mean subthreshold $V_{m}$ from individual neurons overlaid and split by direction of change ( $g$, depolarizing, $n=15 / 23$ neurons; $h$, hyperpolarizing, $n=4 / 23$ neurons). Thick purple line represents population means $\pm 95 \% \mathrm{Cl}$. (i) Cumulative probability of $\Delta V_{m}$ onsets across all significantly modulated L5B projection neurons $(n=19 / 23$ neurons). Ml, movement initiation. (j) Ratio of normalised area under the curve for $V_{m}$ trajectories during miss versus hit trials. Purple symbols represent data from individual neurons, black symbols represent population mean $\pm 95 \%$ Cl. (k) Representative $V_{m}$ traces from a L5B depolarizing (top) and L5B hyperpolarizing (bottom) neuron across multiple trials. Filed purple bars depict hit trials, open purple bars depict miss trials. For clarity, internally generated movements (IGMs) are not shown. (I) Average subthreshold $\Delta V_{m}$ (purple) and firing rate ( $F R$, black) trajectories for the $L 5 B$ enhanced and $L 5 B$ suppressed neurons shown in $(k)$ aligned to movement initiation (MI). Thick lines represent the mean $\pm 95 \% \mathrm{Cl}$. (m) Correlation between movement-related subthreshold $\Delta V_{m}$ and firing rate changes. Colored symbols represent mean $\pm 95 \% \mathrm{Cl}$ from individual neurons, black line is a linear fit to the data (Pearson's $r$ ). (n) Patch-Clamp recording from M1 $F L$ L5B projection neurons during muscimol inactivation targeted to $M T h_{D N / P N}$ - l-Clamp, current clamp, $M 1_{F L}$, forelimb motor cortex. (o) Average subthreshold $\Delta V_{m} \pm 95 \% C l$ from a $\pm 5 B$ projection

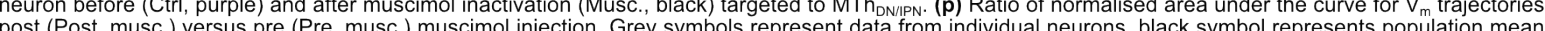
post (Post musc.) versus pre (Pre_musc.) muscimol injection. Grey symbols represent data from individual neurons, black symbol represents population mean 
trajectories and movement initiation. Muscimol inactivation reduced the amplitude and duration of $L 5 B V_{m}$ responses (mean muscimol:control $A U C$ ratio $=0.35,95 \% \mathrm{Cl}[0.26,0.44]$, $\mathrm{n}=3$ neurons from $\mathrm{N}=3$ mice), mirroring $\mathrm{V}_{\mathrm{m}}$ trajectories during miss trials where $\mathrm{MTh}_{\mathrm{DN} / \mathrm{IPN}}$ input is absent (compare Figure 4j and Figure 4p; and Figure 40 and Figure S5h), and reduced task success $($ mean $=0.37$ normalized task success, $95 \% \mathrm{Cl}[0.16,0.59], \mathrm{n}=3$ neurons, $\mathrm{N}$ = 3 mice) (Figure $4 q$ ). Thus, $M T h_{D N / P N}$ input to $M 1_{F L}$ drives activity dynamics necessary for goal-directed movement initiation.

If prior to movement, mice remain in a prepared state awaiting an 'initiation' signal, direct stimulation of the MTh $h_{D N / P N}$ thalamocortical pathway in the absence of the cue could provide an input sufficient to evoke learned movements. We tested this prediction using a dual optogenetic stimulation strategy employing either direct stimulation of $\mathrm{MTh}_{\mathrm{DN} / \mathrm{IPN}}$ neurons or thalamocortical axon terminals in $\mathrm{M} 1_{\mathrm{FL}}$ during the baseline period prior to cue presentation (Figure 5a). By targeting small volumes of AAV2/1-CAG-ChR2 virus to the dorsal-posterior motor thalamus, we restricted opsin expression almost exclusively to neurons in $M_{\text {TT }} \mathrm{D}_{\mathrm{DN} / \mathrm{PN}}$ (Figure S6). Direct stimulation of $M T_{\mathrm{DN} / \mathrm{PN}}$ or axon terminals in $\mathrm{M} 1_{\mathrm{FL}}$ evoked forelimb movements in $9 / 10$ mice, with full lever pushes occurring in $\sim 26 \%$ of trials $\left(\mathrm{MTh}_{\mathrm{DN} / \mathrm{IPN}}\right.$ stimulation, mean $=0.27$ proportion of trials with full lever push, $95 \% \mathrm{Cl}[0.23,0.30], \mathrm{N}=9 / 10$ mice; axon terminal stimulation, mean $=0.25$ proportion of trials with full lever push, $95 \% \mathrm{Cl}$ $[0.13,0.38], \mathrm{N}=6 / 6$ mice, Video $\mathrm{S} 4)$. The duration and reaction times of photostimulated push movements were comparable to cue-evoked trials (Figures $5 b-5 d$ ). In a small proportion of trials, light stimulation evoked partial lever pushes that did not reach the reward zone $\left(\mathrm{MTh}_{\mathrm{DN} / \mathrm{PN}}\right.$ stimulation, mean $=0.15$ proportion of trials, $95 \% \mathrm{Cl}[0.09,0.20], \mathrm{N}=9$ mice; axon terminal stimulation, mean $=0.06$ proportion of trials, $95 \% \mathrm{Cl}[0,0.14], \mathrm{N}=6$ mice) (Figure $5 b)$. Stimulating either pathway in the absence of ChR2 expression did not evoke any detectable forelimb movements $(\mathrm{N}=2)$ (Figure $\mathrm{S6})$. To compare the cellular effects of cueversus ChR2-evoked $M T_{D N / P N}$ input in $M 1_{F L}$, we performed whole-cell recordings from identified $M 1_{F L}$ L5B projection neurons during interleaved cue and $M T h_{D N / P N}$ photostimulation 
a

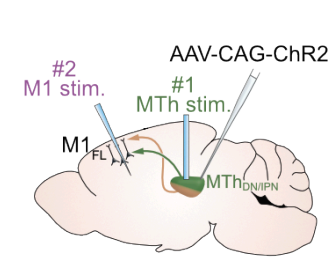

e

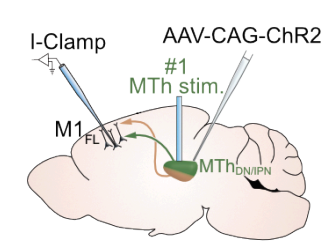

b

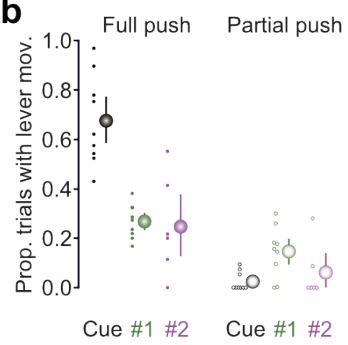

f

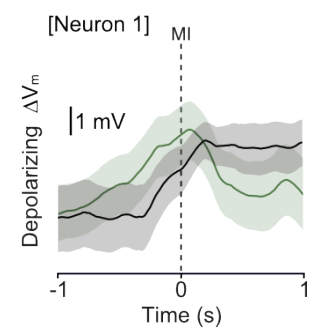

C

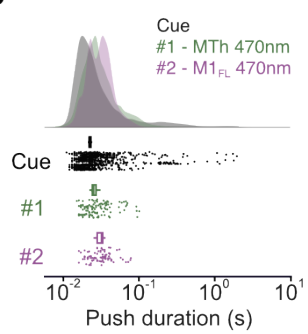

g

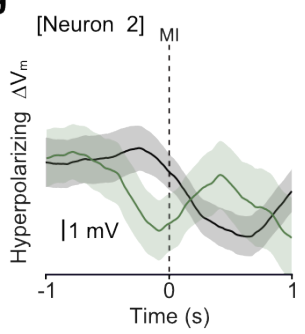

d

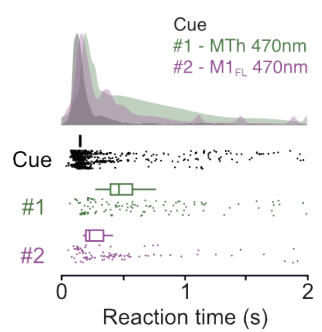

h

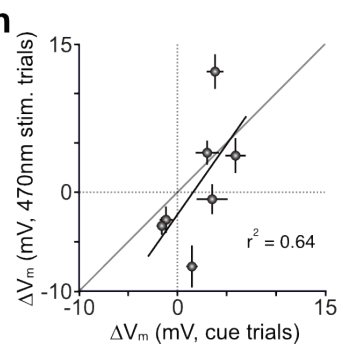

Figure 5. Optogenetic stimulation of $\mathrm{MTh} \mathrm{DN}_{\mathrm{D} / \mathrm{PN}}$ axon terminals recapitulates cued goal-directed movement.

(a) Dual MTh optogenetic stimulation strategy: Channelrhodopsin 2 (ChR2) expression was targeted to neurons in MTh placed directly above $(\# 1)$ or via a tapered optic fiber implanted directly into forelimb motor cortex $\left(\mathrm{M} 1_{\mathrm{FL}}\right)$ (\#2). (b) Comparison of cue-evoked and ChR2-evoked task engagement represented as the proportion of trials with either full (left) or partial (right) lever push movements. Black, cue-evoked; green, direct MTh stimulation; purple, stimulation of $\mathrm{MTh} \mathrm{DN}_{\mathrm{DNN}}$ axon terminals in $\mathrm{M}_{\mathrm{FL}}$. Colored dots represent data from individual mice, colored symbols represent mean $\pm 95 \% \mathrm{Cl}$ For Cue, \#1 and \#2, N = 9, 9 and 6 mice, respectively. (c-d) Raincloud plots showing the distributions of push durations (c) and reaction times (d) of cue-evoked (black) and ChR2-evoked (\#1, green and \#2, purple) hit trials. Box-and-whisker plots of bootstrapped estimates of median statistics. (e) Patch-clamp recording from $M 1_{F \mid}$ L5B projection neurons during ChR2-mediated stimulation of MTh ${ }_{D N / P N}$ neurons. I-Clamp, current clamp; M1 $_{\mathrm{F}}$, forelimb motor cortex. (f-g) Average changes in subthreshold $\mathrm{V}_{m} \pm 95 \% \mathrm{Cl}$ in two L5B projection neurons showing either a pre-movement depolarization (f) or hyperpolarization (g) in response to an auditory cue (black) or ChR2-mediated stimulation of $\mathrm{MTh}_{\text {DNIPN }}$ neurons (green). Dashed line represents movement initiation. (h) Correlation between perimovement cue-evoked and ChR2-evoked subthreshold $\Delta V_{m}$ across $L 5 B$ projection neurons ( $n=7$ neurons, $N=6$ mice). Filled symbols represent mean $\pm 95 \%$

trials (Figure 5e). Remarkably, during photostimulation trials, full push movements were associated with depolarizing or hyperpolarizing $V_{m}$ changes that matched cue-evoked $V_{m}$ changes in the same neuron (Figures $5 f-5 h$ ). Thus, selective recruitment of the $M T h_{D N / P N}-$ $\mathrm{M} 1_{\mathrm{FL}}$ thalamocortical pathway can recapitulate $\mathrm{M} 1_{\mathrm{FL}} \mathrm{L} 5 \mathrm{~B}$ neural activity dynamics required for goal-directed movement initiation.

If behavioral context provides information necessary for learned movement initiation, ChR2evoked forelimb lever push movements should be abolished during direct $M T h_{\text {DN/IPN }}$ stimulation in an altered behavioral context. To test this prediction, we placed trained mice on a flat baseplate in the absence of any support / movable levers, reward spout or water rewards, and compared cue-evoked forelimb movements in both the learned and altered behavioral contexts. Habituation in the altered behavioral context was performed within training session to ensure that the cued lever push behavior was not extinguished. As expected, trained mice generated cue-evoked forelimb lever push trajectories in $63 \%$ of trials in the learned behavioral context (LBC) but in the altered behavioral context (ABC) cue-evoked push-like movements 
bioRxiv preprint doi: https://doi.org/10.1101/802124; this version posted October 16, 2019. The copyright holder for this preprint (which was not certified by peer review) is the author/funder, who has granted bioRxiv a license to display the preprint in perpetuity. It is made available under aCC-BY 4.0 International license.

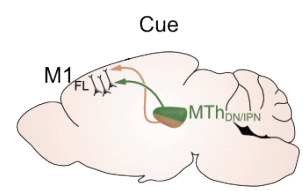

f

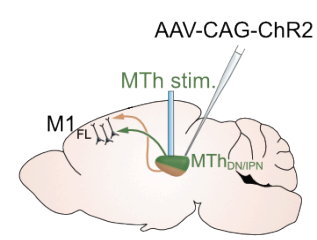

b

Learned behavioural context

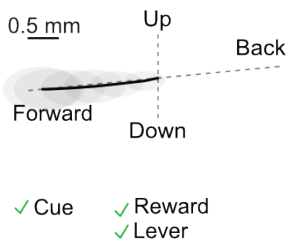

g

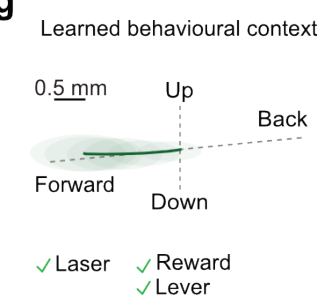

C

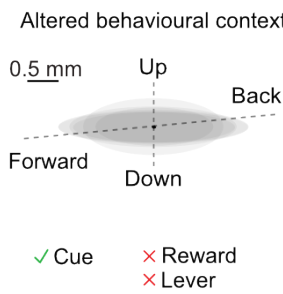

h

Altered behavioural context

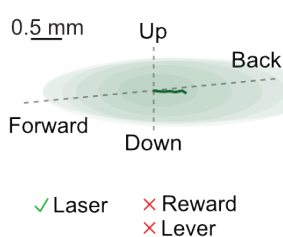

$[0.55,0.71] ; A B C$, mean $=0.02$ (Figures 6a-6e, Video S5). We then replicated the experiment by replacing the auditory cue with direct photostimulation of $M T h_{D N / P N}$ during the baseline period (Figure 6f). If direct $M T h_{D N / P N}$ stimulation alone drives $95 \% \mathrm{Cl}[0.52,0.82] ;$ mean $=0.45$ proportion of trials with push action, $95 \% \mathrm{Cl}[0.40,0.49], \mathrm{N}$ $=3$ mice) (Figures $6 \mathrm{~g}$ and $6 \mathrm{i}$, Video $\mathrm{S} 6$ ). While in the altered behavioral context, direct $M T h_{D N / P N}$ stimulation evoked forelimb movements in $64 \%$ of trials but only $4 \%$ contained pushlike movements (mean $=0.64$ proportion of trials with movement, $95 \% \mathrm{Cl}[0.59,0.69]$; mean $=0.04$ proportion of trials with push action, $95 \% \mathrm{Cl}[0.02,0.07], \mathrm{N}=3$ mice) (Figures $6 \mathrm{~h}$ and 6j, Video S6). Taken together, these results demonstrate that the $M T_{\mathrm{DN} / \mathrm{IPN}}$ thalamocortical

Figure 6. MTh
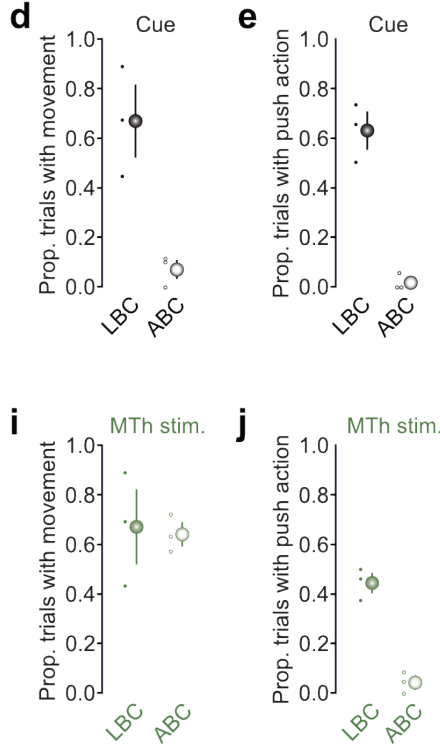

(a) Mouse sagittal brain schematic depicting cue-evoked feedforward input from $M T h_{\mathrm{DN} / \mathrm{PN}}$ to $M 1_{\mathrm{FL}}$. (b-c) Average cue-evoked kinematic forepaw trajectories from an example mouse in a learned behavioral context (i.e. auditory cue, reward spout \& movable lever (b)) or altered behavioral context (i.e. auditory cue, no reward spout $\&$ no movable lever (c)). Thick black line depicts average trajectory overlaid with the $95 \% \mathrm{Cl}$ of frame-by-frame paw position variance. (d-e) Proportion of trials with cue-evoked forelimb movement (d) or forelimb push actions (e) in a learned behavioral context (LBC) versus altered behavioral context (ABC). Colored dots represent data from individual mice, colored symbols represent mean $\pm 95 \% \mathrm{Cl}(\mathrm{N}=3$ mice). (f) MTh was targeted to neurons in MTh mice, colored symbols represent mean $\pm 95 \%$ cll $(\mathrm{N}=3$ mice). was targeted to neurons in $\mathrm{MTh}_{\mathrm{DN} / \mathrm{PN}}$ and stimulated via an optic fiber placed directly above. ( $\mathrm{g}$-h) Average ChR2-evoked kinematic forepaw trajectories from an by-frame paw position variance. (i-j) Proportion of trials with ChR2-evoked forelimb movement (i) or forelimb push actions (j) in a learned behavioral context (LBC) versus altered behavioral context (ABC). Colored dots represent data from individual mice, colored symbols represent mean $\pm 95 \% \mathrm{Cl}$. 
pathway conveys a robust motor timing signal necessary for initiating behavioral contextspecific movement initiation.

\section{Discussion}

Here, we investigated the contribution of the cerebellar-recipient motor thalamocortical pathway to movement initiation, showing that a robust and reproducible feedforward motor timing signal propagating from $M T_{\mathrm{DN} / \mathrm{PN}}$ to $\mathrm{M} 1_{\mathrm{FL}}$ is essential for goal-directed movement initiation. Specifically, we show that trial-to-trial MTh ${ }_{D N / I P N}$ population responses are dominated by a time-locked increase in activity immediately prior to movement initiation that is temporally uncoupled from cue presentation, providing a fixed latency feedforward motor timing signal to $M 1_{F L} \cdot M T h_{D N / P N}$ thalamocortical input is a prerequisite for generating $M 1_{F L}$ layer $5 B$ activity dynamics necessary for movement initiation and blocking MTh $\mathrm{DN}_{\mathrm{D} / \mathrm{PN}}$ output suppresses task engagement. Finally, direct stimulation of $M T h_{D N / P N}$, or their axon terminals in $M 1_{F L}$, in the absence of the cue recapitulated motor cortical activity dynamics and forelimb behavior in the learned behavioral context, but generated semi-random movements in an altered behavioral context where the lever and reward were absent. Together, these data suggest that dentate and interpositus nucleus-recipient motor thalamocortical pathway plays a pivotal role in directly gating movement initiation, thus confirming and extending existing theories of the role of the cerebellar-thalamocortical pathway in initiating goal-directed movement.

By employing population calcium imaging of $M T h_{D N / P N}$ activity we demonstrate that trial-to-trial output from the dentate and interpositus nucleus-recipient regions of motor thalamus do not reflect sensorimotor transformations from cue to movement initiation, instead we suggest that $M T h_{D N / P N}$ output reflects a pure feedforward motor timing signal that indicates the immediate intention to move. In the absence of this input, i.e. local inactivation of MTh to move is blocked resulting in a suppression of goal-directed movement initiation. If the cerebellar-recipient motor thalamocortical pathway conveys a pure motor timing signal, where in the brain is the delay between cue and MTh ${ }_{D N / P N}$ activity onset generated? A signal that 
directly gates movement likely overlaps with preparatory activity in frontal motor regions (Churchland et al., 2006b; Li et al., 2015; Requin et al., 1990) irrespective of how movements are initiated (Lara et al., 2018). Preparatory activity both in frontal motor regions and deep cerebellar nuclei are driven by a cortico-cerebellar loop through the motor thalamus, where persistent neural dynamics across brain regions facilitates movement choice and execution. Thus, sensory-driven persistent activity in frontal motor-associated regions could provide the initial 'decision to move', which propagates through cortico-cerebellar loops to form a discrete motor timing signal at the level of motor thalamus (Chabrol et al., 2019; Churchland et al., 2006a; Gao et al., 2018; Guo et al., 2014). Although our data do not shed light on the origin of the signal, we demonstrate that MTh $\mathrm{DN}_{\mathrm{DN} / \mathrm{PN}}$ is an essential node in the cerebello-cortical loop through which motor timing signals propagate to initiate goal-directed movement. Further studies will be required to determine the contribution of cortico-ponto-cerebellar and cerebellar-thalamocortical loops to sensorimotor transformations across a range of goaldirected motor tasks, and whether information pertaining to movement preparation and execution propagate through the same or parallel subdivisions of the motor thalamus (Chabrol et al., 2019; Gao et al., 2018; Kuramoto et al., 2009; Miller and Brooks, 1982).

The behavioral context-dependence of photoactivated movements suggests that MTh likely conveys a movement-invariant motor initiation signal that converges, at the level of motor cortex, with other long-range inputs necessary for selecting movement type. Consistent with this notion, in the absence of feedforward MTh ${ }_{D N / P N}$ input (i.e. during miss trials or thalamic inactivation), M1 layer 5 projection neurons displayed reduced task-related activity that did not initiate movement. The origin of the convergent long-range input(s) remains unknown, but likely candidates are cortico-cortical interactions between orbitofrontal cortex or frontal motor areas and M1 (Hooks et al., 2013; Reep et al., 1990), thought to accumulate task-relevant information required for motor planning and decision-making (Gao et al., 2018; Li et al., 2015), or basal ganglia-thalamocortical interactions that determine the type, timing and invigoration 
of upcoming movements (Dudman and Krakauer, 2016; Inase et al., 1996; Klaus et al., 2019; Thura and Cisek, 2017; Williams and Herberg, 1987).

Directly activating the MTh $\mathrm{DN}_{\mathrm{IPN}}$ thalamocortical pathway in the altered behavioral context consistently generated semi-random forelimb movements (Tanaka et al., 2018), whereas full recapitulation of the learned behavior could only be achieved in the learned behavioral context. If convergent input from MTh $\mathrm{DN}_{\mathrm{N} / \mathrm{PN}}$ and other task-related brain areas is necessary for learned movement initiation, why can photostimulation of the thalamocortical pathway result in learned movement initiation in the absence of an external sensory cue? Previous studies have shown that behavioral context is an important determinant of neural trajectories during goal-directed motor tasks (Russo et al., 2018; Suresh, 2019), where cortical dynamics evolve in a predetermined manner depending on their initial state (Churchland et al., 2010; Kaufman et al., 2014; Sauerbrei, 2018). In the learned behavioral context, M1 population dynamics likely remain in a quasi-prepared state, awaiting external input to initiate learned forelimb movement. Thus, in some trials (up to $40 \%$ ) direct $M T h_{D N / P N}$ stimulation is likely sufficient to drive M1 neural trajectories towards a state required for learned movement initiation. Conversely, in mice habituated to an unrewarded, altered behavioral context, M1 population dynamics driven by direct $M T h_{D N / I P N}$ stimulation likely evolve from a different initial state resulting in neural trajectories that generate arbitrary, but not goal-directed, forelimb movements (Graziano et al., 2002; Rispal-Padel et al., 1982; Tanaka et al., 2018). A central question for future investigation is how thalamic input and behavioral context contribute to motor cortical population dynamics across learning and different motor behaviors.

In summary, our findings extend our understanding of how specific subdivisions of the mammalian motor thalamus contribute to motor timing (Dormont et al., 1982; Kurata, 2005; Strick, 1976), suggesting that the cerebellar-thalamocortical pathway plays a critical role in the initiation of goal-directed movement. 


\section{References}

Bastian, A.J., and Thach, W.T. (1995). Cerebellar outflow lesions: a comparison of movement deficits resulting from lesions at the levels of the cerebellum and thalamus. Ann. Neurol. 38, 881-892.

Bosch-Bouju, C., Smither, R.A., Hyland, B.I., and Parr-Brownlie, L.C. (2014). Reduced reachrelated modulation of motor thalamus neural activity in a rat model of Parkinson's disease. J. Neurosci. 34, 15836-15850.

Butler, E.G., Horne, M.K., and Hawkins, N.J. (1992). The activity of monkey thalamic and motor cortical neurones in a skilled, ballistic movement. J. Physiol. 445, 25-48.

Chabrol, F.P., Blot, A., and Mrsic-Flogel, T.D. (2019). Cerebellar Contribution to Preparatory Activity in Motor Neocortex. Neuron 103, 506-519 e504.

Churchland, M.M., Cunningham, J.P., Kaufman, M.T., Ryu, S.I., and Shenoy, K.V. (2010). Cortical preparatory activity: representation of movement or first cog in a dynamical machine? Neuron 68, 387-400.

Churchland, M.M., Santhanam, G., and Shenoy, K.V. (2006a). Preparatory activity in premotor and motor cortex reflects the speed of the upcoming reach. J. Neurophysiol. 96, 3130-3146.

Churchland, M.M., Yu, B.M., Ryu, S.I., Santhanam, G., and Shenoy, K.V. (2006b). Neural variability in premotor cortex provides a signature of motor preparation. J. Neurosci. 26, 36973712.

Dormont, J.F., Schmied, A., and Conde, H. (1982). Motor command in the ventrolateral thalamic nucleus: neuronal variability can be overcome by ensemble average. Exp. Brain Res. $48,315-322$.

Dudman, J.T., and Krakauer, J.W. (2016). The basal ganglia: from motor commands to the control of vigor. Current opinion in neurobiology $37,158-166$. 
Fortier, P.A., Kalaska, J.F., and Smith, A.M. (1989). Cerebellar neuronal activity related to whole-arm reaching movements in the monkey. J. Neurophysiol. 62, 198-211.

Gao, Z., Davis, C., Thomas, A.M., Economo, M.N., Abrego, A.M., Svoboda, K., De Zeeuw, C.I., and Li, N. (2018). A cortico-cerebellar loop for motor planning. Nature 563, 113-116.

Gibson, A.R., Houk, J.C., and Kohlerman, N.J. (1985). Magnocellular red nucleus activity during different types of limb movement in the macaque monkey. J. Physiol. 358, 527-549.

Graziano, M.S., Taylor, C.S., and Moore, T. (2002). Complex movements evoked by microstimulation of precentral cortex. Neuron 34, 841-851.

Guo, Z.V., Li, N., Huber, D., Ophir, E., Gutnisky, D., Ting, J.T., Feng, G., and Svoboda, K. (2014). Flow of cortical activity underlying a tactile decision in mice. Neuron 81, 179-194.

Harvey, R.J., Porter, R., and Rawson, J.A. (1979). Discharges of intracerebellar nuclear cells in monkeys. J. Physiol. 297, 559-580.

Holmes, G. (1939). The cerebellum of man. Brain 62, 1-30.

Hooks, B.M., Mao, T., Gutnisky, D.A., Yamawaki, N., Svoboda, K., and Shepherd, G.M. (2013). Organization of cortical and thalamic input to pyramidal neurons in mouse motor cortex. J. Neurosci. 33, 748-760.

Horne, M.K., and Butler, E.G. (1995). The role of the cerebello-thalamo-cortical pathway in skilled movement. Prog. Neurobiol. 46, 199-213.

Inase, M., Buford, J.A., and Anderson, M.E. (1996). Changes in the control of arm position, movement, and thalamic discharge during local inactivation in the globus pallidus of the monkey. J. Neurophysiol. 75, 1087-1104.

Ivry, R.B., and Keele, S.W. (1989). Timing functions of the cerebellum. J. Cog. Neuro. 1, 136152. 
Kaufman, M.T., Churchland, M.M., Ryu, S.I., and Shenoy, K.V. (2014). Cortical activity in the null space: permitting preparation without movement. Nat. Neurosci. 17, 440-448.

Klaus, A., Alves da Silva, J., and Costa, R.M. (2019). What, If, and When to Move: Basal Ganglia Circuits and Self-Paced Action Initiation. Annu. Rev. Neurosci. 42, 459-483.

Kuramoto, E., Furuta, T., Nakamura, K.C., Unzai, T., Hioki, H., and Kaneko, T. (2009). Two types of thalamocortical projections from the motor thalamic nuclei of the rat: a single neurontracing study using viral vectors. Cereb. Cortex. 19, 2065-2077.

Kurata, K. (2005). Activity properties and location of neurons in the motor thalamus that project to the cortical motor areas in monkeys. J. Neurophysiol. 94, 550-566.

Lara, A.H., Elsayed, G.F., Zimnik, A.J., Cunningham, J.P., and Churchland, M.M. (2018). Conservation of preparatory neural events in monkey motor cortex regardless of how movement is initiated. elife 7.

Li, N., Chen, T.W., Guo, Z.V., Gerfen, C.R., and Svoboda, K. (2015). A motor cortex circuit for motor planning and movement. Nature 519, 51-56.

Meyer-Lohmann, J., Hore, J., and Brooks, V.B. (1977). Cerebellar participation in generation of prompt arm movements. J. Neurophysiol. 40, 1038-1050.

Milak, M.S., Shimansky, Y., Bracha, V., and Bloedel, J.R. (1997). Effects of inactivating individual cerebellar nuclei on the performance and retention of an operantly conditioned forelimb movement. J. Neurophysiol. 78, 939-959.

Miller, A.D., and Brooks, V.B. (1982). Parallel pathways for movement initiation of monkeys. Exp. Brain Res. 45, 328-332. 
Mink, J.W., and Thach, W.T. (1991). Basal ganglia motor control. II. Late pallidal timing relative to movement onset and inconsistent pallidal coding of movement parameters. J. Neurophysiol. $65,301-329$.

Mushiake, H., and Strick, P.L. (1993). Preferential activity of dentate neurons during limb movements guided by vision. J. Neurophysiol. 70, 2660-2664.

Ohmae, S., Kunimatsu, J., and Tanaka, M. (2017). Cerebellar Roles in Self-Timing for Suband Supra-Second Intervals. J. Neurosci. 37, 3511-3522.

Person, R.J., Andrezik, J.A., Dormer, K.J., and Foreman, R.D. (1986). Fastigial nucleus projections in the midbrain and thalamus in dogs. Neuroscience 18, 105-120.

Reep, R.L., Goodwin, G.S., and Corwin, J.V. (1990). Topographic organization in the corticocortical connections of medial agranular cortex in rats. J. Comp. Neurol. 294, 262-280.

Requin, J., Lecas, J.C., and Vitton, N. (1990). A comparison of preparation-related neuronal activity changes in the prefrontal, premotor, primary motor and posterior parietal areas of the monkey cortex: preliminary results. Neurosci. Lett. 111, 151-156.

Rispal-Padel, L., Cicirata, F., and Pons, C. (1982). Cerebellar nuclear topography of simple and synergistic movements in the alert baboon (Papio papio). Exp. Brain Res. 47, 365-380.

Rispal-Padel, L., Harnois, C., and Troiani, D. (1987). Converging cerebellofugal inputs to the thalamus. I. Mapping of monosynaptic field potentials in the ventrolateral nucleus of the thalamus. Exp. Brain Res. 68, 47-58.

Russo, A.A., Bittner, S.R., Perkins, S.M., Seely, J.S., London, B.M., Lara, A.H., Miri, A., Marshall, N.J., Kohn, A., Jessell, T.M., et al. (2018). Motor Cortex Embeds Muscle-like Commands in an Untangled Population Response. Neuron 97, 953-966 e958. 
Sakai, S.T., Inase, M., and Tanji, J. (1996). Comparison of cerebellothalamic and pallidothalamic projections in the monkey (Macaca fuscata): a double anterograde labeling study. J. Comp. Neurol. 368, 215-228.

Sauerbrei, B., Guo, J., Mischiati, M., Guo, W., Kabra, M., Verma, N., Branson, K., \& Hantman, A. (2018). Motor cortex is an input-driven dynamical system controlling dexterous movement. BioRxiv 266320; doi: https://doi.org/10.1101/266320.

Schiemann, J., Puggioni, P., Dacre, J., Pelko, M., Domanski, A., van Rossum, M.C., and Duguid, I. (2015). Cellular Mechanisms Underlying Behavioral State-Dependent Bidirectional Modulation of Motor Cortex Output. Cell Reports 26;11(8):1319-30.

Spidalieri, G., Busby, L., and Lamarre, Y. (1983). Fast ballistic arm movements triggered by visual, auditory, and somesthetic stimuli in the monkey. II. Effects of unilateral dentate lesion on discharge of precentral cortical neurons and reaction time. J. Neurophysiol. 50, 1359-1379.

Strick, P.L. (1976). Activity of ventrolateral thalamic neurons during arm movement. J. Neurophysiol. 39, 1032-1044.

Suresh, A.K., Goodman, J. M., Okorokova, E., Kaufman, M. T., Hatsopoulos, N. G. \& Bensmaia, S. J. (2019). Neural population dynamics in motor cortex are different for reach and grasp. BioRxiv 667196; doi: https://doi.org/10.1101/667196.

Tanaka, Y.H., Tanaka, Y.R., Kondo, M., Terada, S.I., Kawaguchi, Y., and Matsuzaki, M. (2018). Thalamocortical Axonal Activity in Motor Cortex Exhibits Layer-Specific Dynamics during Motor Learning. Neuron 100, 244-258 e212.

Thach, W.T. (1975). Timing of activity in cerebellar dentate nucleus and cerebral motor cortex during prompt volitional movement. Brain Res. 88, 233-241. 
Thach, W.T. (1978). Correlation of neural discharge with pattern and force of muscular activity, joint position, and direction of intended next movement in motor cortex and cerebellum. J. Neurophysiol. 41, 654-676.

Thach, W.T. (2013). Does the cerebellum initiate movement. Cerebellum 13, 139-150.

Thura, D., and Cisek, P. (2017). The Basal Ganglia Do Not Select Reach Targets but Control the Urgency of Commitment. Neuron 95(5), 1160-1170.

van Donkelaar, P., Stein, J.F., Passingham, R.E., and Miall, R.C. (2000). Temporary inactivation in the primate motor thalamus during visually triggered and internally generated limb movements. J. Neurophysiol. 83, 2780-2790.

Williams, S.F., and Herberg, L.J. (1987). Motivational vs. motor effects of striatal and pallidal gabergic projections to subthalamic and entopeduncular nuclei, ventromedial thalamus, and ventral globus pallidus. Pharmacol. Biochem. Behav. 26, 49-55.

\section{Acknowledgements}

We are grateful to Tiago Branco, Benjamin Grewe, Jan Gründemann, Matthew Nolan, Gülsen Sürmeli, Brett Mensh and members of the Nolan and Duguid labs for experimental discussions and for comments on the manuscript. Nick Steinmetz for the suggested design of the author contribution matrix. Pseudotyped SAD $\Delta$ G-mCherry(EnvA) rabies virus was a generous gift from Edward Callaway (Salk Institute for Biological Studies) to A.H. AAV-GCaMP6s was a gift from Douglas Kim \& GENIE Project (Addgene 100844-AAV1). pACAGW-ChR2-Venus-AAV was a gift from Karel Svoboda (Addgene plasmid \#20071) and packaged by Christina McClure and Innes Jarmson (Nolan Lab, University of Edinburgh). Confocal microscopy was performed in the IMPACT Imaging Facility at the University of Edinburgh. We thank Marie Zechner for assistance with graphic design. Research was supported by grants from the Biotechnology and Biological Sciences Research Council (BB/R018537/1), DFG fellowship program (SCHI1267/2-1 to J.S. and AM 443/1-1 to J.A.), the Shirley Foundation, Howard Hughes 
Medical Institute (A.H. and C-C.H.) and a Wellcome Senior Research Fellowship $(110131 / Z / 15 / Z)$ to I.D.

\section{Author contributions}

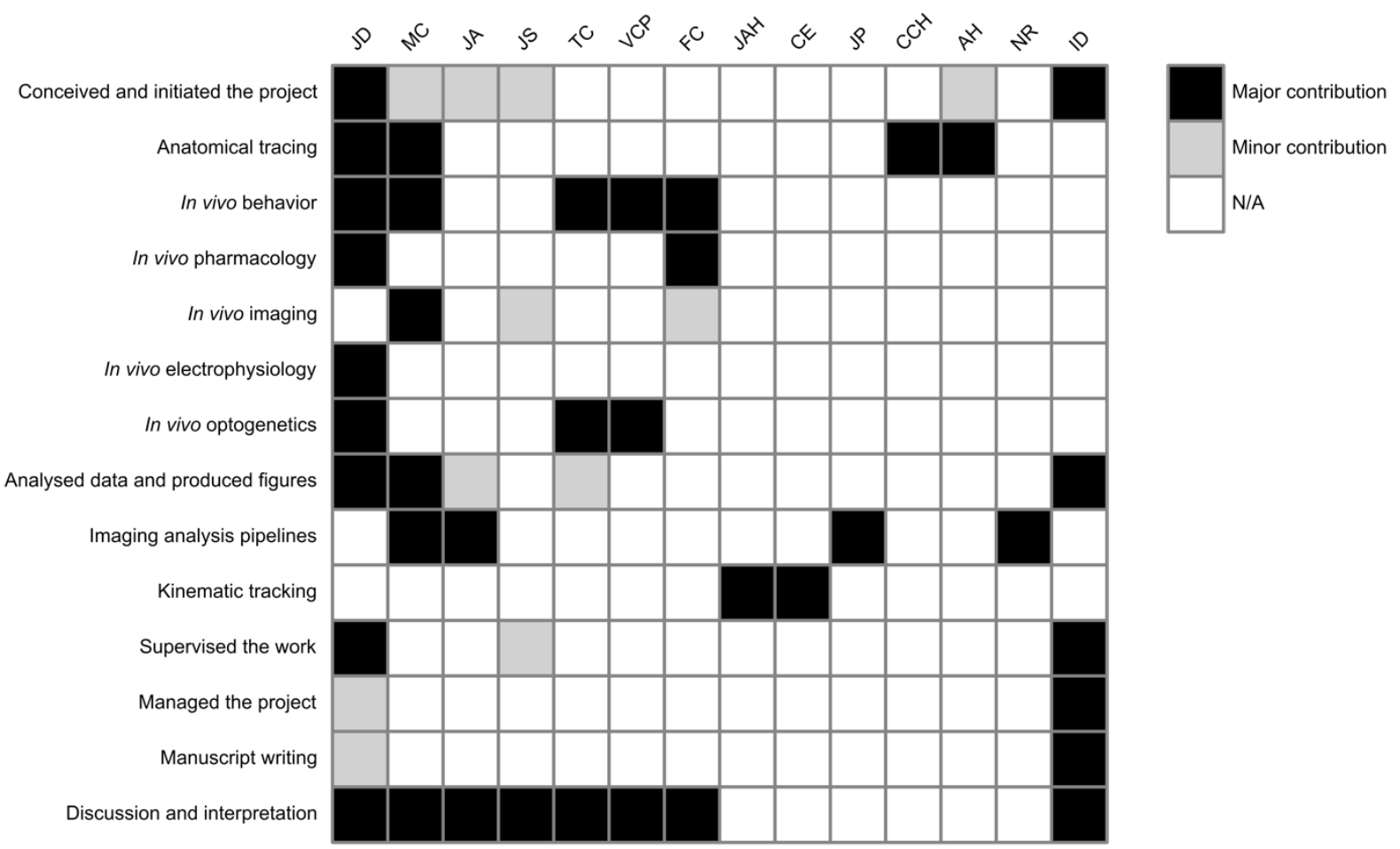

\section{Methods}

\section{Animal husbandry and general surgery}

Male adult C57BL/6J wild-type and Rbp4-Cre (MMRRC, stock 031125-UCD) mice (5-12 weeks old, $20-30 \mathrm{~g}$, one to six animals per cage) were maintained on a reversed 12:12 hour light:dark cycle and provided ad libitum access to food and water. All experiments and procedures were approved by the University of Edinburgh local ethical review committee and performed under license from the UK Home Office in accordance with the Animal (Scientific Procedures) Act 1986. Surgical procedures were performed under $\sim 1.5 \%$ isoflurane anesthesia and each animal received fluid replacement therapy $(0.5 \mathrm{ml}$ sterile Ringer's solution) to maintain fluid balance and buprenorphine $(0.5 \mathrm{mg} / \mathrm{kg})$ for post-operative pain relief. A small lightweight headplate $(0.75 \mathrm{~g})$ was implanted on the surface of the skull using 
cyanoacrylate super glue and dental cement (Lang Dental, USA) and mice were left for 24-48 hours to recover. Craniotomies were performed in a stereotactic frame (Kopf, USA) using a hand-held dentist drill with $0.5 \mathrm{~mm}$ burr (whole-cell patch-clamp recording $\varnothing 300 \mu \mathrm{m}$; viral/tracer/pharmacological compound injection $\varnothing 500-1000 \mu \mathrm{m})$, viral vectors and tracing compounds were delivered via pulled glass pipettes ( $5 \mu \mathrm{l}$, Drummond, $10-20 \mathrm{nl} / \mathrm{min}$ ) using an automated injection system (Model Picospritzer iii, Intracell).

\section{Monosynaptic retrograde rabies tracing}

For monosynaptic retrograde rabies tracing, conditional expression of TVA receptor was achieved by injecting 60nl of AAV2/1-CAG-FLEX-mTagBFP2-2A-TVA $\left(9.0 \times 10^{12}\right.$ genome copies per $\mathrm{ml}(\mathrm{GC} / \mathrm{ml})$ ) into contralateral $\mathrm{M} 1_{\mathrm{FL}}(\mathrm{AP}: 0.6$, $\mathrm{ML}: 1.6$, DV: $-0.7 \mathrm{~mm}$ ) of three Rbp4Cre mice. For anterograde labelling of deep cerebellar nuclei projections to $M M_{D N / P N}, A A V 2 / 1-$ CAG-EGFP $\left(1.1 \times 10^{13} \mathrm{GC} / \mathrm{ml}\right)$ was vertically injected into contralateral dentate (AP: -6.2 , ML: 2.25, DV: $-2.5 \&-2.0 \mathrm{~mm}$ ) and interpositus deep cerebellar nuclei (AP: -6.2, ML: 1.25, DV: 2.5 \& $-2.0 \mathrm{~mm}$ ), with $60 \mathrm{nl}$ injected at each depth. Pseudotyped SAD $\Delta$ G-mCherry(EnvA) rabies virus (produced as previously described (Wickersham et al., 2007; Wickersham et al., 2010) was injected into $M 1_{F L}$ (same coordinates as stated previously) three weeks after the initial injections. Mice were perfused seven days post-rabies virus injection. Sections $(60 \mu \mathrm{m})$ were cut, mounted and imaged at 20x using a Nanozoomer Slide Scanner (Hamamatsu). For all anatomical quantification, raw data images were manually referenced to the Paxinos \& Franklin Mouse Brain Atlas (Paxinos \& Franklin, 2008). The distribution of fluorescence was manually outlined and independently verified.

\section{Conventional retrograde tracing}

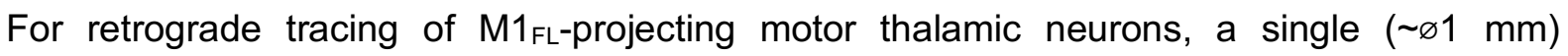
craniotomy was performed above contralateral $\mathrm{M} 1_{\mathrm{FL}}(\mathrm{AP}: 0.6, \mathrm{ML}: 1.6, \mathrm{DV}:-0.7 \mathrm{~mm}$ ), and 150 $\mathrm{nl}$ of red $(590 \mathrm{~nm})$ retrobeads (Lumafluor Inc.) was injected at four points equidistant from the center of the craniotomy. After recovery, mice were returned to the home cage for $\sim 7$ days 
before being anaesthetized with euthatal $(0.10-0.15 \mathrm{ml})$ and transcardially perfused with 30 $\mathrm{ml}$ of ice-cold PBS followed by $30 \mathrm{ml}$ of $4 \%$ paraformaldehyde (PFA) in PBS solution. Brains were post-fixed in PFA overnight at $4^{\circ} \mathrm{C}$ and transferred to $10 \%$ sucrose solution for storage. Coronal sections $(60 \mu \mathrm{m})$ were cut with a vibratome (Leica VT1000S), mounted using Vectashield mounting medium $(\mathrm{H}-1000$, Vector Laboratories), and imaged using a laser scanning confocal microscope (Leica TCS-NT). To assess the density of $\mathrm{M}_{\mathrm{FL}}$-projecting neurons originating in the ventrolateral motor thalamus, 200nl of CTB-Alexa647 (Invitrogen) was injected into $\mathrm{M} 1_{\mathrm{FL}}(\mathrm{AP}: 0.6, \mathrm{ML}: 1.6, \mathrm{DV}:-0.7 \mathrm{~mm}$ ). After 7 days post injection, mice were

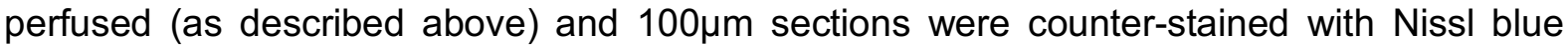
before being imaged with a Leica LSM800 confocal microscope. Cells were counted in a representative $300 \times 300 \mu \mathrm{m}$ region of the ventrolateral motor thalamus and counts were independently verified.

\section{Behavioral training}

After recovery from head plate surgery, mice were handled extensively before being head restrained and habituated to a custom lever push behavioral setup. Mice were trained to perform horizontal lever push movements $(4 \mathrm{~mm})$ in response to a $6 \mathrm{kHz}$ auditory cue in order to obtain a 4-8 $\mu \mathrm{l}$ water reward. To increase task engagement mice were placed on a water control paradigm ( $1 \mathrm{ml} /$ day) and weighed daily to ensure body weight remained above $85 \%$ of baseline. Mice were trained once per day for 30 mins, with a quasi-random inter-trial-interval of 4-6s followed by presentation of an auditory cue. Mice responded within a $10 \mathrm{~s}$ window early in training, reduced to a $2 \mathrm{~s}$ window prior to recording, and were deemed 'expert' after achieving >90 rewards per session on two consecutive days. Lever movements during the ITI would result in a 'lever reset' and commencement of a subsequent ITI.

\section{In vivo pharmacology}

To assess the behavioral effects of $\mathrm{M} 1_{\mathrm{FL}} / \mathrm{MTh}_{\mathrm{DN} / \mathrm{PN}}$ inactivation, the contralateral forelimb was shaved under general anesthesia and the wrist, elbow and shoulder joints were marked with 
black ink. Mice were allowed to recover for at least 60 mins before being head-restrained in the behavioral apparatus. After 5 min of baseline task execution, the lever was locked and a small volume of the $\mathrm{GABA}_{A}$ receptor agonist muscimol (dissolved in external solution containing $150 \mathrm{mM} \mathrm{NaCl}, 2.5 \mathrm{mM} \mathrm{KCl}, 10 \mathrm{mM}$ HEPES, $1.5 \mathrm{mM} \mathrm{CaCl}_{2}$ and $1 \mathrm{mM} \mathrm{MgCl}_{2}$ ) or saline was injected into the target area $\left(\mathrm{M}_{\mathrm{FL}}\right.$ : $200 \mathrm{nl}$ of $2 \mathrm{mM}$ muscimol at each of 5 sites centered on AP: 0.6, ML: 1.6, DV: $-0.7 \mathrm{~mm}$; MTh ${ }_{\mathrm{DN} / \mathrm{IPN}}: 200 \mathrm{nl}$ of $1 \mathrm{mM}$ muscimol, AP: $-1.1, \mathrm{ML}$ : 1.0, DV: $-3.4 \mathrm{~mm}$ ). Mice were randomly assigned to drug or control groups, and experiments performed blinded. To confirm anatomical location of each drug injection, $1 \% \mathrm{w} / \mathrm{v}$ of red (590 $\mathrm{nm}$ ) retrobeads (Lumaflor Inc.) was included in the drug/saline solution. Behavioral metrics were analyzed in 5-minute epochs using a two-way repeated measures ANOVA to determine statistical significance with Bonferroni-Holm correction for multiple comparisons.

\section{GRIN lens imaging}

To perform population calcium imaging in motor thalamus we injected $200 \mathrm{nl}$ of AAV1-SynGCaMP6s $\left(2.9 \times 10^{13} \mathrm{GC} / \mathrm{ml}\right.$, Addgene 100844-AAV1) into contralateral $\mathrm{MTh}_{\mathrm{DN} / \mathrm{IPN}}(\mathrm{AP}:-1.1$, $\mathrm{ML}: 1.0, \mathrm{DV}:-3.4 \mathrm{~mm}$ ) before implanting a lightweight headplate as described above. After 710 days, a gradient-index (GRIN) lens (Grintech NEM-060-15-15-520-S-1.0p; $600 \mu \mathrm{m}$ diameter, $4.83 \mathrm{~mm}$ length, 0.5 numerical aperture) was implanted as described previously (Xu et al., 2016). In brief, a sterile needle (1.1 mm OD) surrounding the GRIN lens was lowered to a depth of $3.2 \mathrm{~mm}$ and subsequently retracted leaving the lens at the desired depth. The lens was then secured in place with UV curing glue (Norland Products, USA) and dental cement (Lang Dental, USA). Fields of view were checked every 14 days for clarity and GCaMP6s expression. After 4-8 weeks mice began water restriction and behavioral training. Two-photon calcium imaging was performed in expert mice during task engagement with a $320 \times 320 \mu \mathrm{m}$ field of view $(600 \times 600$ pixels $)$ at $40 \mathrm{~Hz}$ frame rate, using a Ti:Sapphire pulsed laser (Chameleon Vision-S, Coherent, CA, USA; $<70$ fs pulse width, $80 \mathrm{MHz}$ repetition rate) tuned to $920 \mathrm{~nm}$ wavelength with a $40 x$ objective lens. For confirmation of GRIN lens location and viral expression, mice were perfused as described above and sections $(100 \mu \mathrm{m})$ were cut with 
a vibratome, counterstained with Nissl blue, and imaged using a slide scanner (Zeiss Axioscan). GRIN lens location was determined using the Paxinos \& Franklin Mouse Brain atlas (Paxinos \& Franklin, 2008), and anatomical confirmation within $M M_{\text {DN/PN }}$ was used to determine data inclusion. Motion artefacts in the raw fluorescence videos were corrected using NoRMCorre (Pnevmatikakis et al., 2017). In brief, NoRMCorre performs non-rigid motion correction by splitting each FOV into overlapping patches, estimating the $\mathrm{xy}$ translation for each patch, and upsampling the patches to create a smooth motion field, correcting for nonuniform motion artefacts caused by raster scanning or brain movement. Regions of interest (ROls, polygonal areas) were manually drawn in Fiji (Schindelin et al., 2012) and fluorescence signals were decontaminated and extracted using nmf_sklearn to remove fluorescence originating from neuropil and neighboring cells (Keemink et al., 2018). Normalized fluorescence was calculated as $\Delta \mathrm{F} / \mathrm{F}_{0}$, where $\mathrm{F}_{0}$ was calculated as the 5 th percentile of the $1 \mathrm{~Hz}$ low-pass filtered raw fluorescence signal and $\Delta \mathrm{F}=\mathrm{F}-\mathrm{F}_{0}$. To define early responsive neurons, average $\Delta \mathrm{F} / \mathrm{F}_{0}$ signals during baseline and peri-movement epochs were compared (baseline epoch $=500 \mathrm{~ms}$ pre-cue; movement epoch $=-250$ to +500 ms peri-movement) using a Wilcoxon rank sum test with a significance threshold of $P<0.01$. The direction of the response was defined as suppressed or enhanced by comparing the median value of the $\Delta F / F_{0}$ signal during both epochs. Late responsive suppressed/enhanced neurons were identified by comparing the $500 \mathrm{~ms}$ pre-cue baseline epoch with a 500 ms pre-reward epoch using a Wilcoxon rank sum test with a significance threshold of $P<0.01$. For presentation, movementaligned $\Delta \mathrm{F} / \mathrm{F}_{0}$ signals were smoothed with the loess method using a 40 -frame sliding window and baseline corrected to the mean $\Delta \mathrm{F} / \mathrm{F}_{0}$ during the $500 \mathrm{~ms}$ pre-cue epoch. To investigate the relationship between $\Delta F / F_{0}$ trajectories and reaction time, reaction times were split into thirds (short [0 - $350 \mathrm{~ms}]$, medium [350 - $900 \mathrm{~ms}$ ] and long [>900 ms] and only FOVs with a sufficient number of trials per reaction time category were included in further analysis. The onset times of early enhanced neurons was calculated per trial for each FOV by employing a previously published onset detection algorithm using a slope sum function (SSF) (Zong et al., 2003) with the decision rule and window of the SSF adapted to calcium imaging data 
(threshold $10 \%$ of peak, SSF window 375 ms, smoothed with a Savitzky Golay filter across 27

frames with order 2). To reduce the influence of noisy individual traces biasing onset detection, each onset was determined as the median of 10,000 bootstrap samples. After calculating an onset for each trial, a kernel density estimate was calculated for the mean onset across trials. The area under this mean population kernel density estimate was calculated using numerical trapezoidal integration. The reliability index for each neuron was defined as the mean Pearson's correlation coefficient across pairs of trials in a defined window from -500 to +500 ms peri-movement initiation. The signal-to-noise ratio was defined as the ratio of mean absolute peak $\Delta F / F_{0}$ change (1s pre-cue to 2 s post-movement) and $\Delta F / F_{0} S D$ during the precue baseline. Time-to-half-maximum $\Delta \mathrm{F} / \mathrm{F}_{0}$ was calculated as the time from cue onset to $50 \%$ of the $\Delta F / F_{\text {peak }}$ trial-to-trial.

\section{In vivo electrophysiology}

Whole-cell patch-clamp recordings targeted to layer $5 \mathrm{~B}, 600-950 \mu \mathrm{m}$ from the pial surface, were obtained from awake head restrained mice. Signals were acquired at $20 \mathrm{kHz}$ using a Multiclamp 700B amplifier (Molecular Devices) and filtered at $10 \mathrm{kHz}$ using PClamp 10 software in conjunction with a DigiData 1440 DAC interface (Molecular Devices). No bias current was injected during recordings and the membrane potential was not corrected for junction potential. Resting membrane potentials were recorded immediately after attaining the whole-cell configuration (break-in). Series resistances (Rs) ranged from 23.6 to $45.5 \mathrm{M} \Omega$. Patch pipettes (5.5-7.5 M 2 ) were filled with internal solution (285-295 mOsm) containing: 135 mM K-gluconate, 4 mM KCl, 10 mM HEPES, 10 mM sodium phosphocreatine, 2 mM MgATP, $2 \mathrm{mM} \mathrm{Na}{ }_{2} \mathrm{ATP}, 0.5 \mathrm{mM} \mathrm{Na}{ }_{2} \mathrm{GTP}$, and $2 \mathrm{mg} / \mathrm{ml}$ biocytin ( $\mathrm{pH}$ adjusted to 7.2 with $\mathrm{KOH}$ ). External solution contained: $150 \mathrm{mM} \mathrm{NaCl}, 2.5 \mathrm{mM} \mathrm{KCl}, 10 \mathrm{mM} \mathrm{HEPES}, 1 \mathrm{mM} \mathrm{CaCl}_{2}$, and $1 \mathrm{mM} \mathrm{MgCl}_{2}$ (adjusted to $\mathrm{pH} 7.3$ with $\mathrm{NaOH}$ ). All electrophysiology recordings were analyzed using custom written scripts in MATLAB. Individual action potentials (APs) were detected with a waveletbased algorithm (Nenadic and Burdick, 2005) and AP threshold was defined as the membrane potential $\left(V_{m}\right)$ at maximal $d^{2} V / d^{2}$ up to $3 \mathrm{~ms}$ before AP peak and manually verified. For 
subthreshold $V_{m}$ analysis APs were clipped by removing data points between -1 and $+9 \mathrm{~ms}$ peri-AP threshold. Average AP firing frequencies were calculated by convolving spike times with a $50 \mathrm{~ms}$ Gaussian kernel. Significant changes in subthreshold $V_{m}$ and AP firing frequency were defined by comparing bootstrapped $95 \%$ confidence intervals of mean movementaligned $V_{m}$ and $A P$ frequency trajectories to zero (baseline epoch $=200 \mathrm{~ms}$ pre-cue; movement epoch $=-100$ to +100 ms peri-movement). Mean changes in $V_{m}\left(\Delta V_{m}\right)$ were calculated by subtracting the mean $V_{m}$ during baseline ( $1 \mathrm{~s}$ epoch prior to cue) from the mean $V_{m}$ during peri-movement epoch (-250 to $+250 \mathrm{~ms}$ epoch when aligned to movement onset). All mean $\Delta \mathrm{V}_{\mathrm{m}}$ trajectories were decimated and median filtered with a $50 \mathrm{~ms}$ sliding window. Population mean $\Delta V_{m}$ trajectories were normalized to the largest absolute mean $\Delta V_{m}$ value in a 1.5 second peri-movement window. Peri-movement $\Delta V_{m}$ onsets were detected as the $10 \%$ rise-time of $V_{m}$ trajectories when aligned to movement. To compare subthreshold $V_{m}$ dynamics during hit and miss trials, cue-aligned periods of $V_{m}$ were baseline subtracted and the area under the $\left|\Delta V_{m}\right|$ trajectory from cue onset to median reward delivery was calculated via trapezoidal numerical integration with a $50 \mathrm{~ms}$ sample rate. We calculated the Pearson correlation coefficient between $\Delta \mathrm{V}_{\mathrm{m}}$ and $\Delta$ firing rate for all significantly modulated neurons.

\section{Immunohistochemistry}

To morphologically identify neurons after recording, deeply anesthetized mice were transcardially perfused with $4 \%$ paraformaldehyde. Mouse brains were post-fixed overnight and coronal sections $(60 \mu \mathrm{m})$ of M1 $\mathrm{FL}$ were cut with a vibratome (Leica VT1000 S). For neuron location recovery, sections were incubated in streptavidin AlexaFluor-488 (1:1000, Molecular Probes) in $0.1 \mathrm{M}$ phosphate buffered saline (PBS) containing $0.5 \%$ Triton X-100, mounted (Vectashield, VectorLabs), imaged using a Zeiss LSM 510 Meta confocal microscope (20x objective) and referenced to the Franklin and Paxinos Mouse Brain Atlas (Paxinos \& Franklin, 2008). To identify projection targets of individually recorded neuron (Schiemann et al., 2015), sections were further processed by heat-mediated antigen retrieval in $10 \mathrm{mM}$ sodium citrate buffer ( $\mathrm{pH} \mathrm{6.0)}$ for $3 \mathrm{hrs}$ at $80^{\circ} \mathrm{C}$. Sections were incubated in blocking solution (0.01 M PBS, 
$10 \%$ normal goat serum (v/v), $0.5 \%$ Triton X-100 (v/v)) at $22{ }^{\circ} \mathrm{C}$ for $2 \mathrm{hrs}$ and incubated overnight at $22{ }^{\circ} \mathrm{C}$ in a primary antibody mixture containing mouse monoclonal anti-Satb2 (1:200, Cat. No. ab51502, Abcam) and rat monoclonal anti-Ctip2 (1:1000, Cat. No. ab18465, Abcam) dissolved in carrier solution (0.01 M PBS, $1 \%$ goat serum, $0.5 \%$ Triton X-100). Slices were then incubated overnight at $22{ }^{\circ} \mathrm{C}$ in a secondary antibody mixture containing AlexaFluor568 goat anti-mouse (1:750, Molecular Probes) and AlexaFluor-647 goat anti-rat (1:750, Molecular Probes) dissolved in carrier solution (0.01 M PBS, 1 \% goat serum, $0.5 \%$ Triton X100), mounted and imaged using a Nikon A1R FLIM confocal microscope (Nikon, Europe). Images were analyzed offline using Fiji.

\section{Optogenetic experiments}

For optogenetic activation of $M T_{\mathrm{DN} / \mathrm{PN}}$ neurons or axon terminals in $\mathrm{M} 1_{\mathrm{FL}}, 250 \mathrm{nl}$ of $\mathrm{AAV} 2 / 1$ CAG-GhR2-GFP $\left(4.7 \times 10^{11} \mathrm{GC} / \mathrm{ml}\right.$, Addgene 20071; control virus: AAV2-CAG-mCherry $\left.\left(5.2 \times 10^{11} \mathrm{GC} / \mathrm{ml}\right)\right)$ was injected unilaterally into contralateral MTh $\mathrm{DN}_{\mathrm{DPN}}(\mathrm{AP}:-1.1, \mathrm{ML}: 1.0, \mathrm{DV}$ : -3.4 mm). For direct MTh was implanted $\sim 300 \mu \mathrm{m}$ dorsal to the viral injection site and trains of pulsed $473 \mathrm{~nm}$ light (8 $\mathrm{mW}, 16.6 \mathrm{~Hz}$ pulse frequency, 33.3\% duty cycle) were delivered using a solid-state laser (DPSS, Civillaser, China) and shutter (LS3S2T1, Uniblitz) controlled by an Arduino control system. For direct simulation of MTh Italy) were implanted to a depth of $1 \mathrm{~mm}$ at the center of $\mathrm{M} 1_{\mathrm{FL}}(\mathrm{AP}: 0.6, \mathrm{ML}: 1.6, \mathrm{DV}:-1.0 \mathrm{~mm}$ ) and $12 \mathrm{~mW}, 473 \mathrm{~nm}$ light was delivered with parameters as described above. Prior to optogenetic stimulation experiments, mice were trained to expert level performance and habituated to light emanating from an uncoupled optic fiber and the sound of shutter activation. During recording sessions, mice were exposed to 3 different trial types: (1) cue and shutter; (2) laser and shutter; and (3) shutter only. Trials were presented with the following pattern: 1, $1,3,1,1,2, \ldots$ repeating for 30 minutes. For the majority of mice, direct $M \mathrm{MT}_{\mathrm{DN} / \mathrm{IPN}}$ stimulation was followed by $M T h_{D N / P N}$ neuron axon terminal stimulation in $\mathrm{M}_{\mathrm{FL}}$ on the following day. In some experiments, whole-cell patch-clamp recordings (as described above) were performed 
in combination with direct $M T h_{D N / P N}$ stimulation. To investigate behavioral context, mice which had previously experienced $M T h_{D N / P N}$ stimulation were head restrained above a 3D printed baseplate (Wanhao i3 Duplicator) without support/movable levers or reward spout and habituated to the altered behavioral context for 2 sessions, interleaved with normal training to ensure that the cued goal-directed motor behavior was not extinguished. To compare effects of MTh $\mathrm{DN}_{\mathrm{D} / \mathrm{PN}}$ stimulation in the learned and altered behavioral contexts, mice first underwent a 15 minute optogenetic stimulation protocol in the learned context, then returned to their home cage for 5 mins before being exposed to a 15 minute optogenetic stimulation protocol in the altered behavioral context. For histological confirmation of the injection site and optic fiber placement, mice were perfused and post-fixed for 2 additional days, before tissue slices were collected and imaged as described above. The center of the optic fiber (COF) was defined as the most ventral extent of the optic fiber tract across all slices from each brain as measured from the pial surface. Where tracts of equal depth were present, the coronal section containing the largest diameter tract tip was identified as the COF. The expression of ChR2-Venus was coarsely defined by first referencing three coronal slices (120 $\mu \mathrm{m}$ spacing) centered on the COF to the Franklin \& Paxinos Mouse Brain Atlas (Paxinos \& Franklin, 2008) before manually evaluating the proportion of each of the principle motor thalamic nuclei (AM, anteromedial; VL, ventrolateral; VPM, ventral posteromedial nucleus; VPL, ventral posteromedial; VM, ventromedial) containing fluorescence, and categorizing three levels based on expression covering $0 \%, 0-50 \%$ and $50-100 \%$ of each nucleus. Proportions of push-like movements in cue- and laser- trials were calculated by correcting for the behavioral error rate, i.e. subtracting the proportion of pushes observed in shutter only trials (3) to obtain a lower bound for induced movement proportion. $\Delta \mathrm{V}_{\mathrm{m}}$ trajectories for both cue-evoked and optogenetic stimulationevoked movement trials were calculated as described above, and trial-by-trial $\Delta \mathrm{V}_{\mathrm{m}}$ changes were based on comparing the $200 \mathrm{~ms}$ pre-laser or pre-cue epoch with the $200 \mathrm{~ms}$ perimovement epoch within each trial. We calculated the Pearson correlation coefficient between cue- and ChR2-evoked $\Delta \mathrm{V}_{\mathrm{m}}$ in hit trials. 


\section{Forelimb kinematic tracking}

Behavior from all experimental and habituation days was recorded at 300 frames per second using a high-speed camera (Pharmacological experiments: Genie HM640, Dalsa; optogenetic experiments: Mako U U-029, Allied Vision) and acquired with Streampix 7 (Norpix), synced using a TTL output from the DigiData 1440 DAC interface. Forepaw and wrist positions during pharmacological inactivation experiments were calculated by tracking forepaw markers using a custom written tracking script in Blender (2.79b, Blender Foundation). Contour plots of paw positions densities were calculated during $2 \mathrm{~s}$ epochs prior to cue presentation by sorting the paw positions by distance from the mean and computing 20 increasingly inclusive convex hulls around $5 \%$ portions of the data to define each contour level. Directional tracking of forelimb movement in the learned/altered behavioral context was performed using Deep Lab Cut, a markerless video tracking toolbox (Mathis et al., 2018). Paw trajectories were plotted for the $100 \mathrm{~ms}$ post movement onset epoch in the learned behavioral context (LBC), and for the altered behavioral context $(\mathrm{ABC})$ we plotted trajectories in the epoch $100 \mathrm{~ms}$ after the LBC median reaction time, due to a lack of movement in the majority of $A B C$ trials. Push-like movements were defined as trials with an initial paw trajectory vector between $100^{\circ}$ and $210^{\circ}$. To measure gross forelimb movement, we defined a region-of-interest (ROI) covering the contralateral (left) forelimb and calculated the motion index (MI) for each successive frame $f$ as $M I_{f}=\sum_{i=1}^{N}\left(c_{f+1, i}-c_{f, i}\right)^{2}$, where $c_{f, i}$ is the grayscale level of the pixel $\mathrm{i}$ of the ROI, $\mathrm{N}$ pixels per $\mathrm{ROI}^{32}$. Movement trials were defined by calculating the $\mathrm{Ml}>\theta$ within $500 \mathrm{~ms}$ of cue/laser onset, with the threshold $\theta$ defined as two standard deviations above mean MI.

\section{Statistics}

Data analysis was performed using custom-written scripts in MATLAB 2019a and code will be made available on request. Data are reported as mean $\pm 95 \%$ bootstrapped confidence interval, 10,000 bootstrap samples, unless otherwise indicated. Where multiple measurements were made from a single animal, suitable weights were used to evaluate 
summary population statistics and to obtain unbiased bootstrap samples. Statistical comparisons using the significance tests stated in the main text were made in MATLAB 2019a, and statistical significance was considered when $\mathrm{P}<0.05$ unless otherwise stated. Data were tested for normality with the Shapiro-Wilk test, and parametric/non-parametric tests were used as appropriate and as detailed in the text.

\section{References}

Keemink, S. W. et al. FISSA: A neuropil decontamination toolbox for calcium imaging signals. Sci Rep 8, 3493 (2018).

Mathis, A. et al. DeepLabCut: markerless pose estimation of user-defined body parts with deep learning. Nat Neurosci 21, 1281-1289 (2018).

Nenadic, Z. \& Burdick, J. W. Spike detection using the continuous wavelet transform. IEEE Trans Biomed Eng 52, 74-87 (2005).

Paxinos, G., \& Franklin, K. B. J. The Mouse Brain in Stereotaxic Coordinates, Compact: The Coronal Plates and Diagrams. 3rd Edition, 256 (Elsevier Science Publishing Co Inc, Academic Press Inc, 2008).

Pnevmatikakis, E. A. \& Giovannucci, A. NoRMCorre: An online algorithm for piecewise rigid motion correction of calcium imaging data. J Neurosci Methods 291, 83-94 (2017).

Schindelin, J. et al. Fiji: an open-source platform for biological-image analysis. Nat Methods 9, 676-682 (2012).

Wickersham, I. R., Finke, S., Conzelmann, K. K. \& Callaway, E. M. Retrograde neuronal tracing with a deletion-mutant rabies virus. Nat Methods 4, 47-49 (2007). 
Wickersham, I. R., Sullivan, H. A. \& Seung, H. S. Production of glycoprotein-deleted rabies viruses for monosynaptic tracing and high-level gene expression in neurons. Nat Protoc 5, 595-606 (2010).

Xu C, Krabbe S, Gründemann J, Botta P, Fadok J.P., Osakada F, Saur D, Grewe B.F., Schnitzer M.J., Callaway E.M., Lüthi A. Distinct Hippocampal Pathways Mediate Dissociable Roles of Context in Memory Retrieval. Cell 167(4), 961-972 (2016).

Zong, W., Heldt, T., Moody, G.B. and Mark, R.G. An Open-Source Algorithm to Detect Onset of Arterial Blood Pressure Pulses. Computers in Cardiology 30, 4 (2003). 